\title{
DYNAMICAL MODEL FOR THE ZODIACAL CLOUD AND SPORADIC METEORS
}

\author{
David Nesvorn ${ }^{1}$, Diego JanChes ${ }^{2}$, David Vokrouhlick ${ }^{1,3}$, Petr PokornÝ $^{1,3}$, \\ William F. BottKe ${ }^{1}$, And Peter Jenniskens ${ }^{4}$ \\ ${ }^{1}$ Department of Space Studies, Southwest Research Institute, 1050 Walnut St., Suite 300, Boulder, CO 80302, USA \\ ${ }^{2}$ Space Weather Laboratory, Code 674, GSFC/NASA, Greenbelt, MD 20771, USA \\ ${ }^{3}$ Institute of Astronomy, Charles University, V Holešovičkách 2, CZ-18000, Prague 8, Czech Republic \\ ${ }^{4}$ Carl Sagan Center, SETI Institute, 515 N. Whisman Road, Mountain View, CA 94043, USA \\ Received 2011 May 31; accepted 2011 September 9; published 2011 November 29
}

\begin{abstract}
The solar system is dusty, and would become dustier over time as asteroids collide and comets disintegrate, except that small debris particles in interplanetary space do not last long. They can be ejected from the solar system by Jupiter, thermally destroyed near the Sun, or physically disrupted by collisions. Also, some are swept by the Earth (and other planets), producing meteors. Here we develop a dynamical model for the solar system meteoroids and use it to explain meteor radar observations. We find that the Jupiter Family Comets (JFCs) are the main source of the prominent concentrations of meteors arriving at the Earth from the helion and antihelion directions. To match the radiant and orbit distributions, as measured by the Canadian Meteor Orbit Radar (CMOR) and Advanced Meteor Orbit Radar (AMOR), our model implies that comets, and JFCs in particular, must frequently disintegrate when reaching orbits with low perihelion distance. Also, the collisional lifetimes of millimeter particles may be longer ( $\gtrsim 10^{5} \mathrm{yr}$ at $\left.1 \mathrm{AU}\right)$ than postulated in the standard collisional models $\left(\sim 10^{4} \mathrm{yr}\right.$ at $\left.1 \mathrm{AU}\right)$, perhaps because these chondrule-sized meteoroids are stronger than thought before. Using observations of the Infrared Astronomical Satellite to calibrate the model, we find that the total cross section and mass of small meteoroids in the inner solar system are $(1.7-3.5) \times 10^{11} \mathrm{~km}^{2}$ and $\sim 4 \times 10^{19} \mathrm{~g}$, respectively, in a good agreement with previous studies. The mass input required to keep the zodiacal cloud in a steady state is estimated to be $\sim 10^{4}-10^{5} \mathrm{~kg} \mathrm{~s}^{-1}$. The input is up to $\sim 10$ times larger than found previously, mainly because particles released closer to the Sun have shorter collisional lifetimes and need to be supplied at a faster rate. The total mass accreted by the Earth in particles between diameters $D=5 \mu \mathrm{m}$ and $1 \mathrm{~cm}$ is found to be $\sim 15,000$ tons $\mathrm{yr}^{-1}$ (factor of two uncertainty), which is a large share of the accretion flux measured by the Long Term Duration Facility. The majority of JFC particles plunge into the upper atmosphere at $<15 \mathrm{~km} \mathrm{~s}^{-1}$ speeds, should survive the atmospheric entry, and can produce micrometeorite falls. This could explain the compositional similarity of samples collected in the Antarctic ice and stratosphere, and those brought from comet Wild 2 by the Stardust spacecraft. Meteor radars such as CMOR and AMOR see only a fraction of the accretion flux ( $\sim 1 \%-10 \%$ and $\sim 10 \%-50 \%$, respectively), because small particles impacting at low speeds produce ionization levels that are below these radars' detection capabilities.
\end{abstract}

Key words: comets: general - meteorites, meteors, meteoroids - zodiacal dust

\section{INTRODUCTION}

The zodiacal cloud (ZC) is a circumsolar disk of small debris particles produced by asteroid collisions and comets. Nesvorný et al. (2010, hereafter N10) developed a dynamical model for particle populations released by asteroids and comets, and used the model to determine the relative contribution of asteroid and cometary material to the ZC. They found that the mid-infrared (MIR) emission from particles produced in the asteroid belt is mostly confined to within latitudes $b \lesssim 30^{\circ}$ of the ecliptic. Conversely, the $\mathrm{ZC}$ has a broad latitudinal distribution so that strong thermal emission is observed even in the direction to the ecliptic poles (e.g., Hauser et al. 1984; Kelsall et al. 1998). This shows that asteroidal particles can represent only a small fraction of the ZC.

Based on a comparison of the model with observations of the Infrared Astronomical Satellite (IRAS), N10 proposed that $\gtrsim 90 \%$ of the ZC's emission at MIR wavelengths comes from dust grains released by Jupiter Family Comets (JFCs), and $\lesssim 10 \%$ comes from the Oort Cloud Comets (OCCs), HalleyType Comets (HTCs), and/or asteroid collisions. In addition, it was found that the mass input required to keep the ZC in a steady state largely exceeds the mass loss in JFCs due to their normal activity (e.g., Reach et al. 2007). To resolve this problem, N10 suggested that the dominant mass fraction is supplied to the ZC by spontaneous disruptions/splittings of JFCs (e.g., Fernández 2005; Di Sisto et al. 2009).

N10's model implies that the orbits of small meteoroids (diameters $D \lesssim 100 \mu \mathrm{m}$ ) released by JFCs become significantly circularized by Poynting-Robertson (P-R) drag before they can reach $a \sim 1 \mathrm{AU}$ and contribute to the Earth impact record. These particles, at the time of their accretion by the Earth, should thus have relatively low impact speeds $\left(v<20 \mathrm{~km} \mathrm{~s}^{-1}\right)$, low eccentricities, and $a \sim 1$ AU. The large JFC particles ( $D \gtrsim 1 \mathrm{~mm})$, on the other hand, should have a broader distribution of impact speeds, large eccentricities, and $a \sim$ 2-4 AU, mainly because they have presumably short collisional lifetimes (Grun et al. 1985, hereafter G85), and disrupt before they can significantly evolve by P-R drag. As we discuss below, these results appear to be at odds with the observations of sporadic meteors.

Meteors are produced by small interplanetary particles also known as the meteoroids. Based on meteor data, the meteoroids can be divided into two groups: sporadic meteoroids and meteoroid streams. The meteoroid streams are prominent concentrations of particles with similar orbits (Whipple 1951). They are thought to be produced by particles released by active and recently (less than a few thousand years ago) disrupted comets 

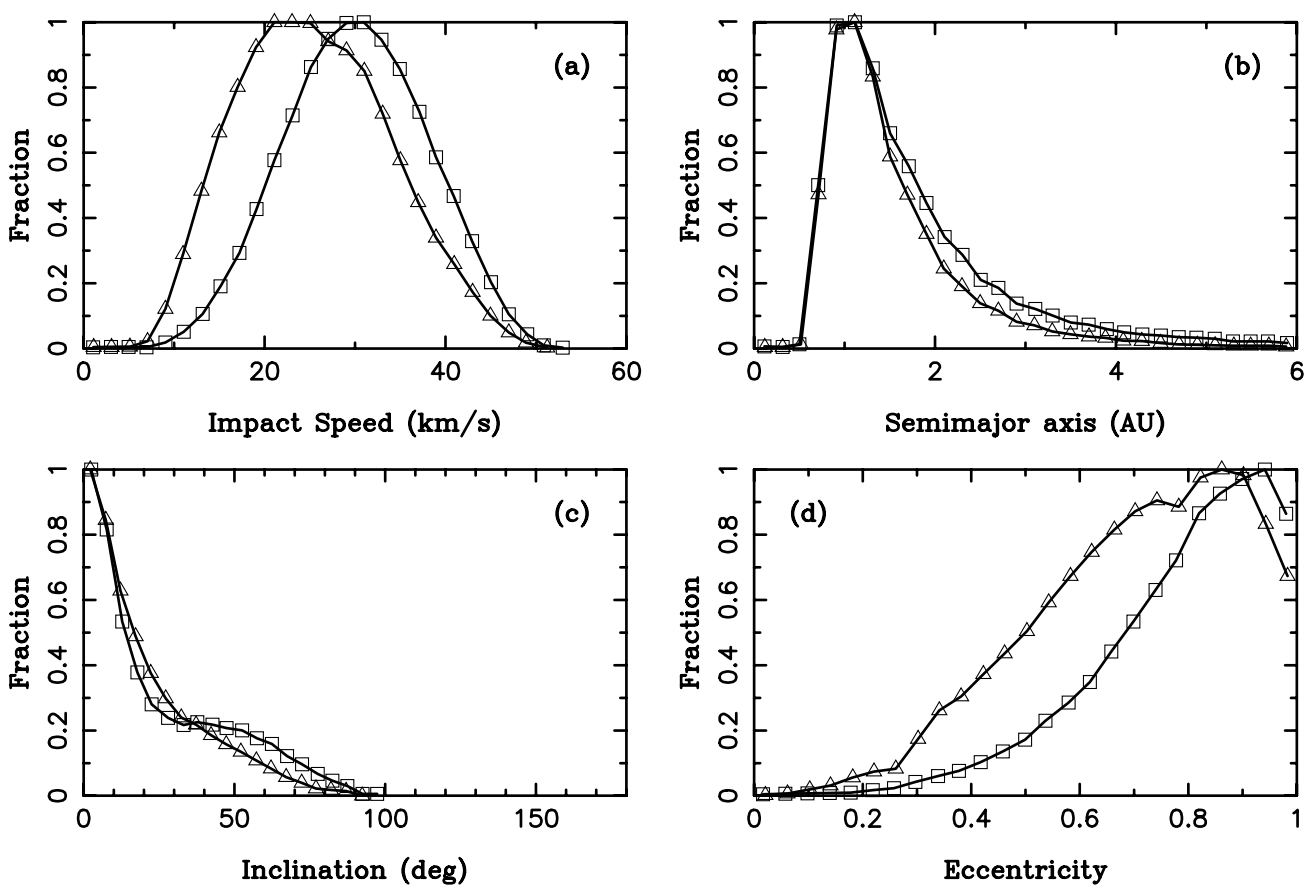

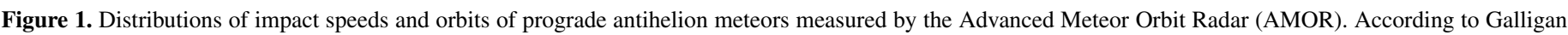

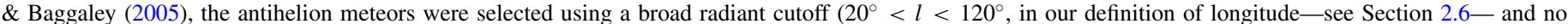

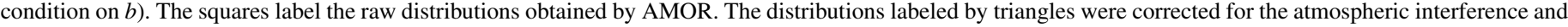

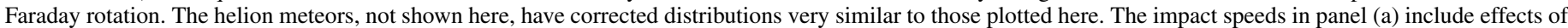

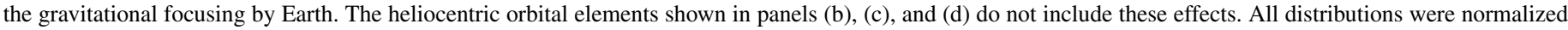
to reach 1 at their maximum. Adapted from Figure 8 in Galligan \& Baggaley (2005).

(Jenniskens 2008). Sporadic meteoroids are those particles that have evolved significantly from their parent body so that they are no longer easily linked to that parent, or to other meteoroids from the same parent. Notably, the time-integrated flux of meteors at Earth is dominated by about a factor of $\sim 10$ by sporadics (Jones \& Brown 1993).

The radiant distribution of sporadic meteors shows several concentrations on the sky known as the helion/antihelion, north/south apex, and north/south toroidal sources (e.g., Campbell-Brown 2008, and the references therein). The prominent helion/antihelion source is the concentration of meteors near the helion and antihelion directions. These meteors are believed to originate from the same population of meteoroids. The two groups differ in impact direction because some particles will impact before their perihelion passage, thus producing meteors with the antihelion radiants, while others will impact after their perihelion passage, producing meteors with the helion radiants.

The helion/antihelion meteoroids have a measured impact speed distribution that peaks at $v \simeq 20-30 \mathrm{~km} \mathrm{~s}^{-1}, a \sim 1 \mathrm{AU}$ with a tail to $3 \mathrm{AU}$ and beyond, $e>0.3$, and low inclinations (Figure 1). Wiegert et al. (2009, hereafter W09) developed a dynamical model to explain these observations. They found that particles released by JFCs, mainly by $2 \mathrm{P} /$ Encke, provide the best match to the observed properties of the helion/antihelion source (see also Jones et al. 2001). ${ }^{5}$

Comet $2 \mathrm{P} /$ Encke has an orbit that is quite unique among JFCs ( $a=2.2 \mathrm{AU}, e=0.85, i=11.8)$, because its aphelion distance lies well within Jupiter's orbit $(Q=a(1+e)=4.1 \mathrm{AU})$. The orbit is relatively stable as it is not affected by close encounters

\footnotetext{
5 The north/south apex meteors are most likely produced by meteoroids released from retrograde HTCs and/or OCCs (Jones et al. 2001; W09; Nesvorný et al. 2011). The origin of the toroidal source is unknown.
}

with Jupiter. ${ }^{6}$ In addition, the comet has a very low perihelion distance $(q=a(1-e)=0.34 \mathrm{AU})$ and is expected to fall into the Sun in $10^{5}-10^{6}$ years (Levison \& Duncan 1994), if it physically survived that long. Finally, 2P/Encke is the source of several meteor streams known as Taurids (Whipple 1939), suggesting, as argued in W09, that it can also be an important source of sporadic meteoroids at 1 AU.

The difference between W09 and N10 lies, in part, in different assumptions on the initial distribution of meteoroids. In W09, the meteoroids launched from $2 \mathrm{P} /$ Encke initially had a low perihelion distance so that even after having evolved by $\mathrm{P}-\mathrm{R}$ drag to $a \sim 1 \mathrm{AU}$, they still retained a relatively large eccentricity. In N10, on the other hand, most meteoroids were released with $q \sim 2.5 \mathrm{AU}$, and greater effects of P-R drag were thus required for particles to reach $1 \mathrm{AU}$. In addition, N10 did not properly include the detection efficiency of meteor radars in their model. This is a central issue, because most meteor radars are only capable of detecting the relatively large and/or fast meteoroids, and may thus produce measurements of the Earth accretion flux that are heavily biased by their detection capabilities.

The agreement between the W09 model and observations of helion/antihelion meteors is not perfect. For example, the W09 model produced tightly clustered distributions of $v$ and $e$ about $v=30 \mathrm{~km} \mathrm{~s}^{-1}$ and $e=0.85$, and lacked orbits with $a \gtrsim 2.5 \mathrm{AU}$ (Figure 2 in W09). The observed speeds and eccentricities have larger spreads, perhaps indicating that the helion/antihelion meteors are produced not by one, but many parent comets with a broad distribution of orbits, including those with $a \gtrsim 2.5$ AU. The inclination distribution produced in W09

\footnotetext{
6 The dynamical origin of $2 \mathrm{P} /$ Encke has yet to be explained, but probably requires non-gravitational forces produced by jets of material escaping from the comet's surface, and gravitational perturbations from the terrestrial planets (Valsecchi 1999).
} 


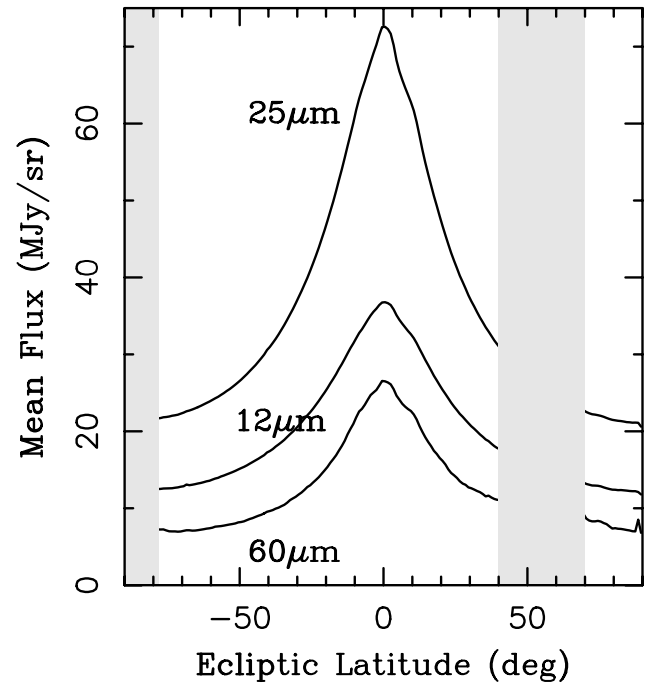

Figure 2. Mean ZC profiles obtained by IRAS in 12, 25, and $60 \mu \mathrm{m}$ wavelengths. To make these profiles, the selected $I R A S$ scans obtained with the $\simeq 90^{\circ}$ solar elongation were centered at the ecliptic, smoothed by a low-pass filter, and combined together (see N10 for details). The gray rectangles at $b<-78^{\circ}$ and $40^{\circ}<b<70^{\circ}$ block the latitude range where the mean fluxes were significantly affected by the galactic plane emission. We do not use the excluded range in this work. The uncertainties of the mean flux values are not shown here; they are too small to clearly appear in the plot. IRAS observations at $100 \mu \mathrm{m}$, not shown here, are less useful for probing the thermal radiation of dust particles in the inner solar system, because of the strong interference with the galactic and extragalactic emission at these wavelenghts.

does not have the resolution needed for a careful comparison with data, but it also seems to be narrower than the observed distribution.

While 2P/Encke, or orbitally similar comets, can be an important source for helion/antihelion meteors, comet 2P/Encke itself cannot be a single dominant source of the ZC. This is because studies of the ZC indicate that $\sim 1000 \mathrm{~kg} \mathrm{~s}^{-1}$ of material need to be injected into interplanetary space to keep the ZC in a steady state (e.g., Leinert et al. 1983, N10). Also, according to $\mathrm{N} 10$, the present mass of the inner $\mathrm{ZC}$ at $<5 \mathrm{AU}$ is $\sim 1-2 \times 10^{19} \mathrm{~g}$, which is roughly equivalent to that of a $25 \mathrm{~km}$ diameter body. For comparison, the mass loss in comet $2 \mathrm{P} /$ Encke is only $\sim 26 \mathrm{~kg} \mathrm{~s}^{-1}$, based on observations of its dust trail (Reach et al. 2007), and the diameter of the nucleus is $\simeq 4.8 \mathrm{~km}$ (Fernández et al. 2000; Boehnhardt et al. 2008).

According to N10, the dominant mass fraction is supplied to the ZC by spontaneous disruptions/splittings of JFCs. Since meteoroids in the $\mathrm{ZC}$ are also expected to produce meteors, the meteor observations discussed above can place important constraints on the ZC's origin. To take advantage of these constraints, and motivated by the results discussed above, we modify N10's model to include a $q$-dependent meteoroid production rate, ${ }^{7}$ and account for the detection efficiency of meteor surveys. We also improve N10's model to consider a

\footnotetext{
7 Direct evidence for JFC disruptions at small heliocentric distances comes from the comparison of dynamical models of JFCs, which follow their transport from the trans-Neptunian region to the inner solar system, with observations (e.g., Levison \& Duncan 1997; Di Sisto et al. 2009). If the radial density of JFCs expected from the dynamical model, assuming no disruptions, is normalized so that it matches the observed (complete) sample of active comets with $q \simeq 1.5 \mathrm{AU}$, it becomes apparent that the model density profile drops far too slowly for $q<1.5 \mathrm{AU}$ to match observations. This means that the comets with small $q$ values must disappear, due to physical effects, more quickly than those with large $q$ values. To match the observed profile, Di Sisto et al. (2009) suggested that the disruption probability of JFCs scales with $q$ as $q^{-\zeta}$, where $\zeta \simeq 0.5-1$.
}

continuous Size Frequency Distribution (SFD) of particles, and more precisely parameterize their collisional disruption in space. We show that, with these modifications of the N10 model, the results match available constraints. We describe the new model in Section 2. The results are reported in Section 3. We estimate the ZC's cross section and mass, meteoroid production rate required to keep the $\mathrm{ZC}$ in a steady state, and the terrestrial accretion rate of interplanetary dust.

\section{MODEL}

Our model includes the following parts: (1) particles of different sizes are released from JFCs (Section 2.1), (2) their orbits evolve under the influence of gravitational and radiation forces (Section 2.2), (3) some particles are thermally or collisionally destroyed (Section 2.3), (4) while in space, particles emit thermal radiation (Section 2.4), which (5) is detected by a telescope observing at MIR wavelengths (Section 2.5), and (6) a small fraction of the initial particle population is accreted by the Earth, producing meteors (Section 2.6). We describe components (1)-(6) below.

Procedures described in Sections 2.5 and 2.6 are mainly required because the raw particle distributions obtained from our numerical integrations of orbits in Section 2.2 do not have a sufficient resolution. We use analytical methods to enhance the resolution in a way that is suitable for (5) and (6).

\subsection{Initial Orbits}

We only consider JFCs in this paper, because previous works showed that they are the main source of the ZC particles and helion/antihelion meteors (e.g., Jones et al. 2001; W09; N10). The asteroid meteoroids have low impact speeds and are not detected by meteor radars. The meteoroids released from longperiod comets contribute to apex meteors and are not modeled here (see Nesvorný et al. 2011 for a discussion of apex meteors).

The orbital distribution of JFCs was taken from Levison \& Duncan (1997, hereafter LD97), who followed the orbital evolution of bodies originating in the Kuiper belt as they are scattered by planets, and evolve in small fractions into the inner solar system. For each critical perihelion distance, $q^{*}$, we selected bodies from LD97's simulations when they reached $q<q^{*}$ for the first time. Particles were released from these source orbits. ${ }^{8}$ We used 10 values of $q^{*}$ equally spaced between $0.25 \mathrm{AU}$ and $2.5 \mathrm{AU}$, particles with $D=10,30,100$, 300,1000 , and $3000 \mu \mathrm{m}$, which should cover the interesting range of sizes, and particle density $\rho=2 \mathrm{~g} \mathrm{~cm}^{-3}$. Our tests show that this size resolution is adequate because the orbital dynamics of, say, a $D=150 \mu \mathrm{m}$ particle is similar to that of a $D=100 \mu \mathrm{m}$ particle. The results for a continuous range of sizes were obtained by interpolation. Varying particle density has only a small effect on their orbital dynamics. Ten thousand particles were released for each $q^{*}$ and $D$ for the total of 0.6 million of the initial orbits.

Upon their release from the parent object, particles will feel the effects of radiation pressure. These effects can be best described by replacing the mass of the Sun, $m_{\odot}$, by $m_{\odot}(1-\beta)$,

\footnotetext{
8 Note that this method does not properly account for the possibility that JFCs lose mass gradually by recurrent splitting events. For example, Di Sisto et al. (2009) assumed that the splitting events occur with certain frequency, considered to be a free parameter, and that a fixed mass fraction, also a free parameter, is lost in each event. Such a model may be physically more appropriate, but has more free parameters.
} 
with $\beta$ given by

$$
\beta=5.7 \times 10^{-5} \frac{Q_{\mathrm{pr}}}{\rho s}
$$

where the particle's radius $s=D / 2$ and $\rho$ are in cgs units. Pressure coefficient $Q_{\mathrm{pr}}$ can be determined using the Mie theory (Burns et al. 1979). We set $Q_{\mathrm{pr}}=1$, which corresponds to the geometrical optics limit, where $s$ is much larger than the incident-light wavelength. Note that all particles considered here are large enough to stay on bound heliocentric orbits after their release (see, e.g., Nesvorný et al. 2011).

In Section 3, we combine the results obtained with different $q^{*}$ and $D$ to mimic the continuous distributions $d N(q)$ and $d N(D)$. This is different from N10 where the source particle distributions were parameterized by the "fading time," particle production rate was assumed to be $q$-independent, and $d N(D)$ was approximated by single size. Here we consider $d N(q)$ and $d N(D)$ that can be approximated by simple power laws. For $d N(q)$, we thus have $d N(q) \propto q^{\gamma} d q$, where $\gamma$ is a free parameter. For $d N(D)$, we have $d N(D)=N_{0} D^{-\alpha} d D$, where $N_{0}$ is a normalization constant and $\alpha$ is the usual slope index (at source). Alternatively, we use the two-slope SFD with $d N(D) \propto D^{-\alpha_{1}} d D$ for $D<D^{*}$ and $d N(D) \propto D^{-\alpha_{2}} d D$ for $D>D^{*}$, where $\alpha_{1}, \alpha_{2}$, and $D^{*}$ are free parameters.

Parameter $\gamma$ can be inferred from the number of JFCs found at each $q$, and their disruption probability as a function of $q$. If the former can be approximated by $q^{\xi}$, where $\xi \sim 0.5$ (LD97; Di Sisto et al. 2009), and the latter is proportional to $q^{-\zeta}$, where $\zeta \simeq 0.5-1$ (Di Sisto et al. 2009), it would be expected that $\gamma=\xi-\zeta \sim-0.5-0$. We use $\gamma=0$ as our starting value, and test the sensitivity of results for $\gamma<0$ and $\gamma>0$. As for $d N(D)$, we set $\alpha \sim 4$ for the whole size range, as motivated by meteor radar observations (e.g., Galligan \& Baggaley 2004), or $D^{*} \sim 100 \mu \mathrm{m}, \alpha_{1}<3$ and $\alpha_{2}>4$, as motivated by space impact experiments (e.g., Love \& Brownlee 1993). Given the various uncertainties of these measurements (see, e.g., Mathews et al. 2001), we also test $D^{*}<100 \mu \mathrm{m}$ and $D^{*}>100 \mu \mathrm{m}$.

\subsection{Orbit Integration}

The particle orbits were numerically integrated with the swift_rmvs3 code (Levison \& Duncan 1994), which is an efficient implementation of the Wisdom-Holman map (Wisdom \& Holman 1991) and which, in addition, can deal with close encounters between particles and planets. The radiation pressure and P-R drag forces were inserted into the Keplerian and kick parts of the integrator, respectively. The change to the Keplerian part was trivially done by substituting $m_{\odot}$ by $m_{\odot}(1-\beta)$. We assumed that the solar-wind drag force has the same functional form as the P-R term and contributes by $30 \%$ to the total drag intensity.

The code tracks the orbital evolution of a particle that revolves around the Sun and is subject to the gravitational perturbations of seven planets (Venus to Neptune; the mass of Mercury was added to the Sun) until the particle impacts a planet, is ejected from the solar system, evolves to within $0.05 \mathrm{AU}$ from the Sun, or the integration time reaches 5 Myr. We removed particles that evolved to $R<0.05 \mathrm{AU}$, because the orbital period for $R<0.05$ AU was not properly resolved by our oneday integration time step. ${ }^{9}$ The particle orbits were recorded at $1000 \mathrm{yr}$ time intervals to be used for further analysis.

\footnotetext{
9 We tested an integration time step of 0.3 day. The results were essentially identical to those obtained with the one-day time step.
}

\subsection{Physical Effects}

The solar system meteoroids can be destroyed by collisions with other particles and by solar heating that can lead to sublimation and vaporization of minerals. Here we explain how we parameterize these processes in our model.

The JFC particles will rapidly lose their volatile ices. We do not model the volatile loss here. The remaining grains will be primarily composed from amorphous silicates and will survive down to very small heliocentric distances. Following Moro-Martín \& Malhotra (2002), Kessler-Silacci et al. (2007), and others, we adopt a simple criterion for the silicate grain destruction. We assume that they are thermally destroyed (sublimate, vaporize) when the grain temperature reaches $T \geqslant$ $1500 \mathrm{~K}$.

Using the optical constants of amorphous pyroxene of approximately cosmic composition (Henning \& Mutschke 1997), we find that a dark $D \gtrsim 100 \mu \mathrm{m}$ grain at $R$ has the equilibrium temperature within $10 \mathrm{~K}$ of a black body, $T \simeq 280 / \sqrt{R} \mathrm{~K}$. According to our simple destruction criterion, $T \geqslant 1500 \mathrm{~K}$, the silicate grains should thus be removed when reaching $R \lesssim 0.035 \mathrm{AU}$. On the other hand, the smallest particles considered in this work ( $D=10 \mu \mathrm{m}$ ) have $T=1500 \mathrm{~K}$ at $R \simeq 0.05 \mathrm{AU}$. Thus, we opted for a simple criterion where particles of all sizes were instantly destroyed and were not considered for statistics, when they reached $R \leqslant 0.05$ AU. Note that, by design, this limit is the same as the one imposed by the integration time step (Section 2.2).

The collisional lifetime of meteoroids, $\tau_{\text {coll }}$, was taken from G85. It was assumed to be a function of particle mass, $m$, and orbital parameters, mainly $a$ and $e$. For example, for a circular orbit at $1 \mathrm{AU}$, particles with $D=100 \mu \mathrm{m}$ and $1 \mathrm{~mm}$ have $\tau_{\text {coll }}^{*}=1.5 \times 10^{5} \mathrm{yr}$ and $7.3 \times 10^{3} \mathrm{yr}$, respectively, where $\tau_{\text {coll }}^{*}$ denotes the collisional lifetime from G85. Also, $\tau_{\text {coll }}^{*}$ increases with $a$. To cope with the uncertainty of the G85's model, we introduced a free parameter, $S$, so that $\tau_{\text {coll }}=S \tau_{\text {coll }}^{*}$. Values $S>1$ increase $\tau_{\text {coll }}$ relative to $\tau_{\text {coll }}^{*}$, as expected, for example, if particles were stronger than assumed in G85, or if the measured impact fluxes were lower (e.g., Dikarev et al. 2005; Drolshagen et al. 2008). See Nesvorný et al. (2011) for a fuller description.

Collisional disruption of particles was taken into account during processing the output from the numerical integration described in Section 2.2. To account for the stochastic nature of breakups, we determined the breakup probability $p_{\text {coll }}=$ $1-\exp \left(-h / \tau_{\text {coll }}\right)$, where $h=1000 \mathrm{yr}$ is the output interval, and $\tau_{\text {coll }}$ was computed individually for each particle's orbit. The code then generated a random number $0 \leqslant x \leqslant 1$ and eliminated the particle if $x<p_{\text {coll }}$.

We caution that our procedure does not take into account the small debris fragments that are generated by disruptions of larger particles. Instead, all fragments are removed from the system. This is an important approximation whose validity needs to be tested in the future.

\subsection{Thermal Emission of Particles}

Meteoroids were assumed to be isothermal, rapidly rotating spheres. The absorption was assumed to occur into an effective cross section $\pi s^{2}$, and emission out of $4 \pi s^{2}$. The infrared flux density (per wavelength interval $d \lambda$ ) per unit surface area at distance $r$ from a thermally radiating particle with radius $s$ is

$$
F_{\lambda}=\epsilon(\lambda, s) B(\lambda, T) \frac{s^{2}}{r^{2}}
$$


where $\epsilon$ is the emissivity and $B(\lambda, T)$ is the energy flux at $(\lambda, \lambda+d \lambda)$ per surface area from a black body at temperature $T$ :

$$
B(\lambda, T)=\frac{2 \pi h c^{2}}{\lambda^{5}}\left[e^{h c / \lambda k T}-1\right]^{-1}
$$

In this equation, $h=6.6262 \times 10^{-34} \mathrm{~J} \mathrm{~s}$ is the Planck constant, $c=2.99792458 \times 10^{8} \mathrm{~m} \mathrm{~s}^{-1}$ is the speed of light, and $k=1.3807 \times 10^{-23} \mathrm{~J} \mathrm{~K}^{-1}$ is the Boltzmann constant.

Since our model does not include detailed emissivity properties of dust grains at different wavelengths, we set the emissivity at $25 \mu \mathrm{m}$ to be 1 and fit for the emissivities at 12 and $60 \mu \mathrm{m}$. We found that the relative emissivities at 12 and $60 \mu \mathrm{m}$ that match the data best are $0.70-0.75$ and $0.95-1$, respectively. Such a variability of MIR emissivity values at different wavelengths is expected for small silicate particles with some carbon content. $T(R)$ was set to be $280 / \sqrt{R} \mathrm{~K}$, as expected for dark $D \gtrsim 10 \mu \mathrm{m}$ particles. See Nesvorný et al. (2006) for a more precise treatment of $\epsilon(\lambda, s)$ and $T(R)$ for dust grains composed of different materials.

\subsection{MIR Observations}

To compare our results with IRAS observations illustrated in Figure $2,{ }^{10}$ we developed a code that models thermal emission from distributions of orbitally evolving particles and produces infrared fluxes that a space-borne telescope would detect depending on its location, pointing direction and wavelength. See Nesvorný et al. (2006) for a detailed description of the code.

In brief, we define the brightness integral along the line of sight of an infrared telescope (defined by fixed longitude $l$ and latitude $b$ of the pointing direction) as

$$
\int_{a, e, i} \text { dadedi } \int_{0}^{\infty} d r r^{2} \int_{D} d D F_{\lambda}(D, r) N(D ; a, e, i) K(R, L, B)
$$

where $r$ is the distance from the telescope, $F_{\lambda}(D, r)$ is the infrared flux (evaluated at the effective wavelength of the telescope's system) per unit surface area at distance $r$ from a thermally radiating particle with diameter $D . K(R, L, B)$ defines the spatial density of particles in the Sun-centered coordinates as a function of $R$, ecliptic longitude, $L$, and latitude, $B$. $N(D, a, e, i)$ is the number of particles having effective diameter $D$ and orbits with $a, e$, and $i$.

We evaluate the integral in Equation (4) by numerical renormalization (see Nesvorný et al. 2006). $F_{\lambda}(D, r)$ is calculated as described in Section 2.4. $N(D, a, e, i)$ is obtained from our numerical simulations (Section 2.2). $K(R, L, B)$ uses analytic expressions for the spatial distribution of particles with fixed $a$, $e$, and $i$, and randomized orbital longitudes (Kessler 1981).

We assume that the telescope is located at $\left(x_{t}=r_{\mathrm{t}} \cos \phi_{\mathrm{t}}, y_{t}=\right.$ $\left.r_{\mathrm{t}} \sin \phi_{\mathrm{t}}, z_{\mathrm{t}}=0\right)$ in the Sun-centered reference frame with $r_{\mathrm{t}}=1 \mathrm{AU}$. Its viewing direction is defined by a unit vector with components $\left(x_{\mathrm{v}}, y_{\mathrm{v}}, z_{\mathrm{v}}\right)$. In Equation (4), the pointing vector can be also conveniently defined by longitude $l$ and latitude $b$ of the pointing direction, where $x_{\mathrm{v}}=\cos b \cos l, y_{\mathrm{v}}=\cos b \sin l$, and $z_{\mathrm{v}}=\sin b$. We fix the solar elongation $l_{\odot}=90^{\circ}$, so that

\footnotetext{
10 We use IRAS because it is the data set we are best familiar with (see Nesvorný et al. 2006; N10). Other, more modern MIR surveys such as the Cosmic Background Explorer (COBE; e.g., Kelsall et al. 1998) have better precision and resolution, but their results do not differ in important ways from those obtained by IRAS. The COBE measurements of the extended MIR emission were used to calibrate the IRAS fluxes as described in Nesvorný et al. (2006).
}

$l=\phi_{\mathrm{t}}+90^{\circ}$, and calculate the thermal flux of various particle populations as a function of $b$ and wavelength. The model brightness profiles at 12,25 , and $60 \mu \mathrm{m}$ are then compared with the mean IRAS profiles shown in Figure 2.

\subsection{Model for Meteor Radar Observations}

We used the Öpik theory (Öpik 1951) to estimate the expected terrestrial accretion rate of JFC particles in our model. Wetherill (1967), and later Greenberg (1982), improved the theory by extending it more rigorously to the case of two eccentric orbits. Here we used a computer code that employs Greenberg's formalism (Bottke et al. 1994). ${ }^{11}$

We modified the code to compute the radiants of the impacting particles. In doing so we properly accounted for all impact configurations and weighted the results by the probability with which each individual configuration occurs, including focusing. The radiants were expressed in the coordinate system, where longitude $l$ was measured from the Earth's apex in counterclockwise direction along the Earth's orbit, and latitude $b$ was measured relative to the Earth's orbital plane. Note that our definition of longitude differs from the one more commonly used for radar meteors, where the longitude is measured from the helion direction. The radiants were calculated before the effects of gravitational focusing were applied.

The meteor radars use different detection methods (i.e., trail versus echo) ${ }^{12}$ and have different sensitivities. Their detection efficiency is mainly a function of the particle's mass and speed, but it also depends on a number of other parameters discussed, for example, in Janches et al. (2008). Following W09, we opt for a simple parameterization of the radar sensitivity function, where the detection is represented by the ionization function

$$
I(m, v)=\frac{m}{10^{-4} \mathrm{~g}}\left(\frac{v}{30 \mathrm{~km} \mathrm{~s}^{-1}}\right)^{3.5} .
$$

All meteors with $I(m, v) \geqslant I^{*}$ are assumed to be detected in our model, while all meteors with $I(m, v)<I^{*}$ are not detected. The ionization cutoff $I^{*}$ is taken to be different for different radars. For example, $I^{*} \sim 1$ for the Canadian Meteor Orbit Radar (CMOR; Campbell-Brown 2008) and $I^{*} \sim 0.01-0.001$ for Advanced Meteor Orbit Radar (AMOR; Galligan \& Baggaley 2004, 2005). For reference, a JFC particle with $v=30 \mathrm{~km} \mathrm{~s}^{-1}$ and $m=10^{-6} \mathrm{~g}$, corresponding to $D \simeq 100 \mu \mathrm{m}$ with $\rho=2 \mathrm{~g} \mathrm{~cm}^{-3}$, has $I(m, v)=0.01$, i.e., a value intermediate between the two thresholds. These meteoroids would thus be detected by AMOR, according to our definition, but not by CMOR. The particle size detection threshold is shown, as a function of $v$, in Figure 3.

To study the orbital properties of different meteor sources, these sources need to be isolated. This is typically done by selecting meteors with specific radiants. To test how the radiant cutoff affects the results, we select the helion meteors with $-90^{\circ}<l<-45^{\circ}$ and $-30^{\circ}<b<30^{\circ}$, and antihelion meteors with $45^{\circ}<l<90^{\circ}$ and $-30^{\circ}<b<30^{\circ}$. Since our code computes the same impact speed and orbit distributions for

\footnotetext{
11 The Öpik theory cannot properly account for the capture of particles in orbital resonances (e.g., Dermott et al. 1994; Šidlichovský \& Nesvorný 1994). Testing the effect of orbital resonances on particles released by JFCs is left for future work.

12 Note that the parameterization described here applies to the specular meteor radars, which detect the meteor trails. A similar parameterization can be developed, however, for the more sensitive High Power and Large Aperture (HPLA) Radars (Fentzke \& Janches 2008; Fentzke et al. 2009) that detect meteor head echoes.
} 


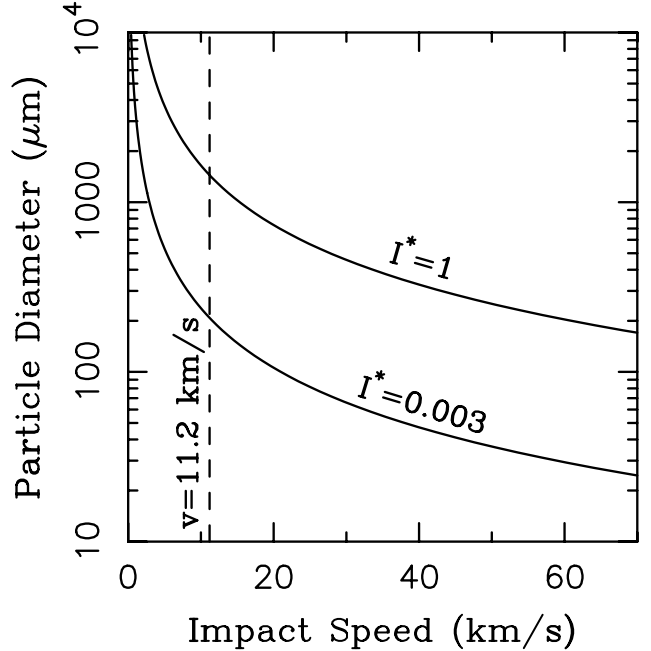

Figure 3. Detection size threshold as a function of the meteor impact speed. All particles above the solid lines are assumed to be detected. The thresholds are $I^{*} \simeq 1$ for $\mathrm{CMOR}$ and $I^{*} \simeq 0.003$ for AMOR. Meteors occur to the right from the dashed vertical line that denotes the Earth's escape speed $\left(v_{\mathrm{esc}}=11.2 \mathrm{~km} \mathrm{~s}^{-1}\right)$.

the helion and antihelion sources, we combine the results from the two radiant cutoffs together. Note, therefore, that our method cannot capture the suspected asymmetry between the helion and antihelion sources (see, e.g., W09, and the references therein).

\section{RESULTS}

We performed hundreds of tests with the model described in Section 2. The main parameters of these tests were the (1) size distribution of JFC particles at the source, as defined by $D^{*}, \alpha_{1}$ and $\alpha_{2},(2)$ power index of the initial perihelion distribution, $d N(q) \propto q^{\gamma} d q$, and (3) collisional lifetime of particles, $\tau_{\text {coll }}$. To compare our model with meteor observations, we specified the appropriate ionization threshold and applied the usual $\chi^{2}$ statistics (see, e.g., Nesvorný et al. 2006). To simplify the presentation of results, we first discuss selected cases that illustrate the trends with different parameters. These cases are generally representative for a wide range of parameter values, as explained in the following text.

\subsection{AMOR}

We start by discussing the results relevant to AMOR, because AMOR is capable of detecting particles with $D \sim 100 \mu \mathrm{m}$ (Figure 3), and can thus provide constraints on the particle sizes that are thought to be dominant in the ZC. Figure 4 shows the distributions of impact speeds and orbits of JFC meteoroids for $D^{*}=100 \mu \mathrm{m}, \gamma=0, S=1$, and several values of the ionization cutoff. With $I^{*}=0$, corresponding to no cutoff on mass or impact speed, the impact speed distribution, $d N(v)$, is strongly peaked toward the Earth's escape speed $\left(v_{\text {esc }}=11.2 \mathrm{~km} \mathrm{~s}^{-1}\right)$. When $I^{*}=0.003$ cutoff is applied, as roughly expected for the AMOR detections, $d N(v)$ has a maximum at $v \simeq 25 \mathrm{~km} \mathrm{~s}^{-1}$. This illustrates the crucial importance of the ionization cutoff for the interpretation of meteor radar observations.

Given the strong effect of the ionization cutoff it is difficult to imagine how the radar observations can be correctly "debiased," based solely on the measurements, corrections, and considerations of the Earth-impact probability of different orbits (e.g., Taylor \& McBride 1997; Galligan \& Baggaley 2004; Campbell-Brown 2008), to obtain the real distribution of meteoroids at $1 \mathrm{AU}$. As shown in Figure 4, the real distribution can be very different from the observed one; although meteors at low speeds may dominate the real distribution, only a tiny fraction are detected. This highlights the importance of dynamical modeling.
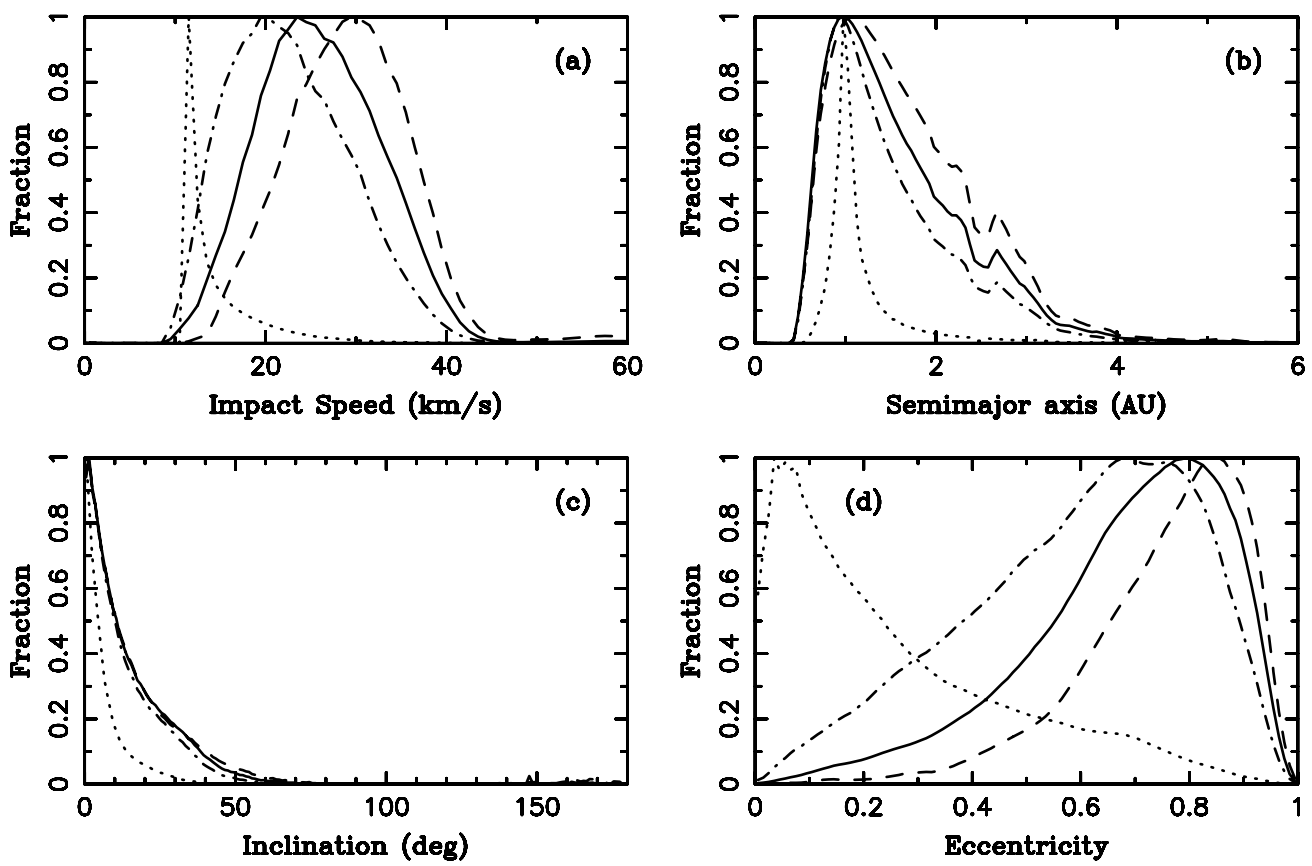

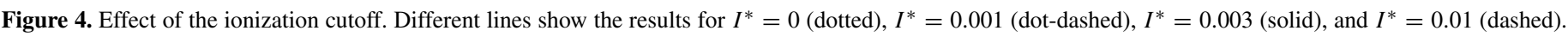

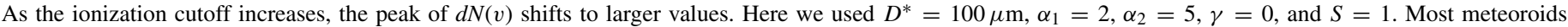

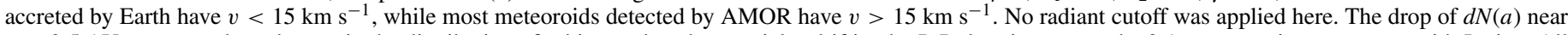

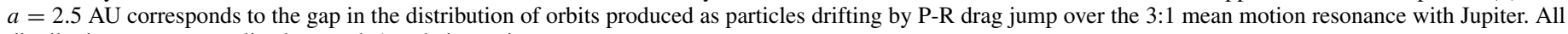
distributions were normalized to reach 1 at their maximum. 

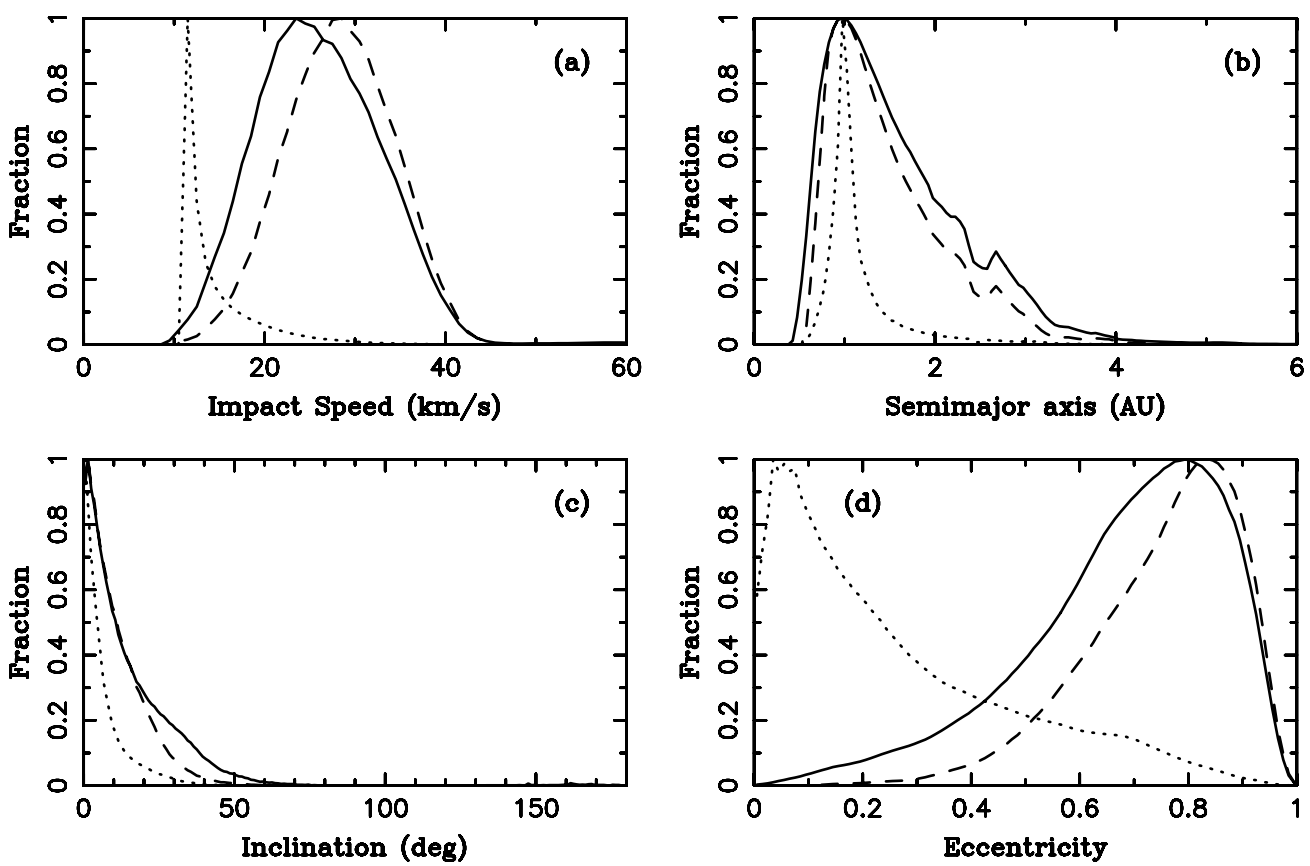

Figure 5. Effect of the radiant cutoff. Different lines show the results for $I^{*}=0$ without a radiant cutoff (dotted), $I^{*}=0.003$ without a radiant cutoff (solid), and $I^{*}=0.003$ with radiant cutoff (dashed). As in Figure 4, we used $D^{*}=100 \mu \mathrm{m}, \alpha_{1}=2, \alpha_{2}=5, \gamma=0$, and $S=1$.

In a similar fashion, the observed eccentricity distribution, $d N(e)$, is strongly biased toward large values by the ionization cutoff, while the underlying distribution has more small and moderate values (Figure 4(d)). This explains, at least in part, why N10 were unable to obtain meteor-like $d N(e)$, because they did not model the meteor detection in detail. The model semimajor axis and inclination distributions, $d N(a)$ and $d N(i)$, are also strongly affected by the ionization cutoff. With $I^{*}=0.003$, both distributions become significantly broader than those computed for $I^{*}=0$ (Figures 4(b) and (c)).

The distribution of impact speeds obtained in our model has a peak value of $v=20 \mathrm{~km} \mathrm{~s}^{-1}$ for $I^{*}=0.001$ and $v=30 \mathrm{~km} \mathrm{~s}^{-1}$ for $I^{*}=0.01$, in good agreement with the AMOR measurements of helion/antihelion meteors that show a peak at $v=20-25 \mathrm{~km} \mathrm{~s}^{-1}$. The spread of model $d N(v)$ (Figure 4(a)), however, is slightly narrower than the one indicated by observations (Figure 1(a)). We will discuss this difference later in this section and show that it can be related to the initial SFD of particles produced by JFCs.

Figure 5 illustrates the effect of the radiant cutoff for $I^{*}=$ 0.003 and the case described above. The radiant cutoff, as defined in Section 2.6, moves $d N(v)$ to slightly larger values (Figure 5(a)) and leads to narrower distributions of $a, e$, and $i$. More aggressive radiant selection criteria, such as the ones used in Campbell-Brown (2008) to define the helion/antihelion sources, would produce a slightly larger effect. On the other hand, Galligan \& Baggaley (2005) adopted a very broad radiant cutoff $\left(-120^{\circ}<l<-30^{\circ}\right.$ and $20^{\circ}<l<120^{\circ}$, respectively, for our definition of $l$, and no condition on $b$ ). According to our tests, these broad selection criteria give results that are similar to those with no radiant cutoff.

The effect of the radial distribution of initial orbits is illustrated in Figure 6. As expected, $\gamma<0$ produces $d N(v)$ that peaks at larger values, and $d N(e)$ that is more skewed toward $e=1$. This is because more particles are produced with small $q$ values in this case, and these particles tend to have larger $v$ and $e$ values when they impact. The effects of $\gamma>0$ are opposite to those of $\gamma<0$. Interestingly, $d N(a)$ and $d N(i)$ obtained with $I^{*}=0.003$ are not very sensitive to changes of $\gamma$.

Together with Figure 4, these results show that it can be difficult to constrain the value of $\gamma$ from the fits to the AMOR measurements alone, because the effects of $\gamma$ are similar to those produced by slight changes in the detection efficiency, and can be confused with them. A detailed knowledge of the instrument sensitivity, that goes beyond the simple concept of the ionization cutoff described in Section 2.6, will be required for a more constrained modeling (see, e.g., Fentzke \& Janches 2008; Fentzke et al. 2009).

The effects of initial $d N(D)$ of particles, as discussed below, are in many ways similar to those produced by changes of $\gamma$ and $I^{*}$. Figure 7 illustrates the effect of $D^{*}$. Again, $d N(v)$ and $d N(e)$ show more variation than $d N(a)$ and $d N(i)$. While for $D^{*}=30 \mu \mathrm{m}$, the velocity peak shifts to $v \simeq 30 \mathrm{~km} \mathrm{~s}^{-1}$, it moves to $v \simeq 20 \mathrm{~km} \mathrm{~s}^{-1}$ for $D^{*}=300 \mu \mathrm{m}$. This variation can be linked to the ionization cutoff. For example, with $D^{*}=30 \mu \mathrm{m}$, particles tend to be smaller and will be detected with $I^{*}=0.003$ only if their speeds are larger.

The distributions $d N(v)$ shown in Figures 4(a)-7(a) are all slightly narrower than the one indicated by observations (see Figure 1(a)). This difference cannot be resolved by varying $\gamma, S$, or $I^{*}$. Instead, to resolve this problem, we needed to assume that the power index of $d N(D)$ is $3 \lesssim \alpha \lesssim 4$, at least in the size range relevant to AMOR observations. To illustrate this case, Figure 8 shows the distributions for $\alpha=\alpha_{1}=\alpha_{2}=3.5$. While $d N(a)$, $d N(e)$ and $d N(i)$ have not changed much relative to Figure 4 , the new distribution $d N(v)$ with $I^{*}=0.01-0.001$ becomes broader, thus better mimicking the AMOR measurements.

This trend can be easily understood. With a sharp SFD break at $D^{*} \sim 100 \mu \mathrm{m}$, the particles that produce most meteors with $I>0.01-0.001$ are those with $D \sim 100 \mu \mathrm{m}$. These particles have similar orbital histories and produce a relatively narrow $d N(v)$. With $\alpha_{1}=\alpha_{2} \sim 3.5$, on the other hand, the size range of particles significantly contributing to AMOR meteors increases, relative to the previous case, producing a larger variability in 

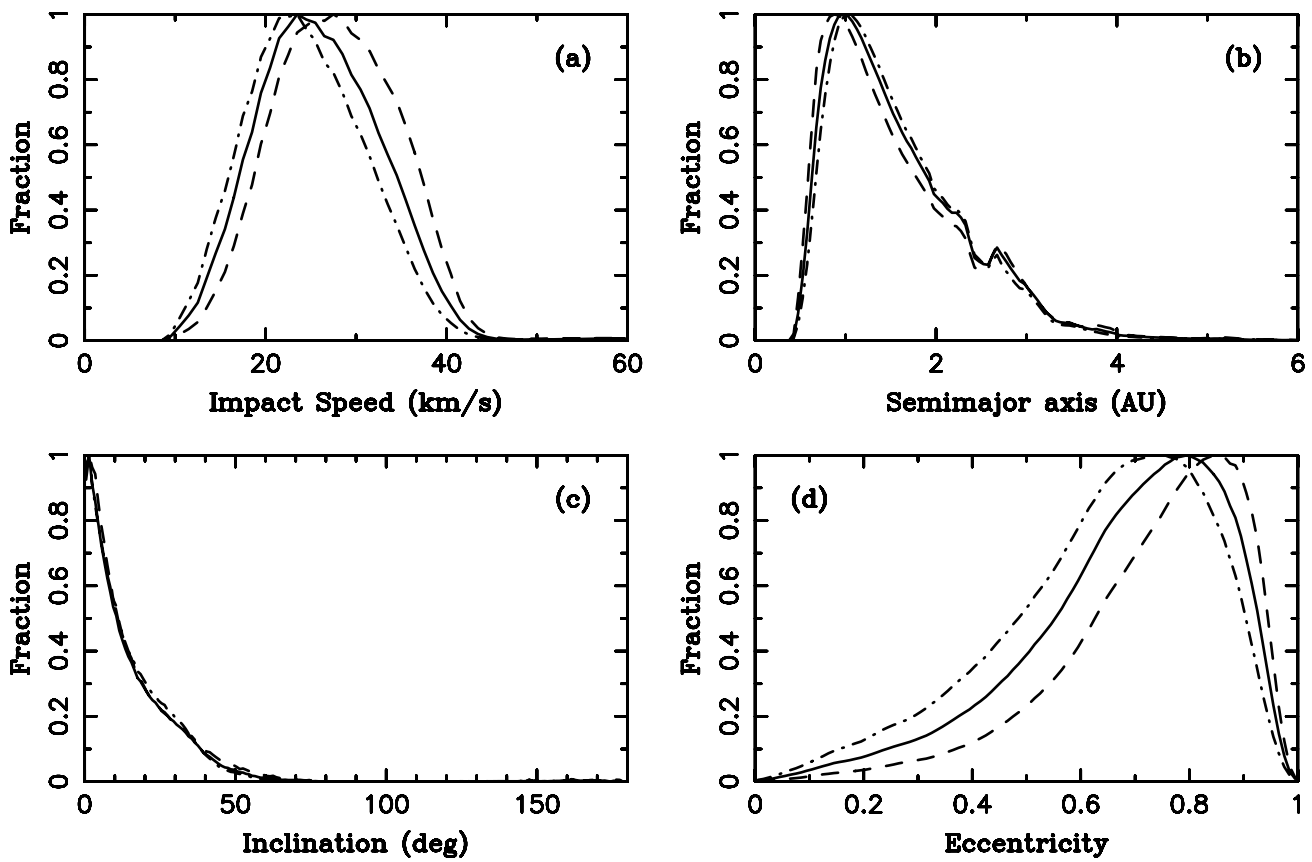

Figure 6. Effect of $\gamma$. Different lines show the results for $\gamma=0$ (solid), $\gamma=-1$ (dashed), and $\gamma=1$ (dot-dashed). As in Figure 5, we used $I^{*}=0.003, D^{*}=100 \mu$ m, $\alpha_{1}=2, \alpha_{2}=5$, and $S=1$. No radiant cutoff was applied here.
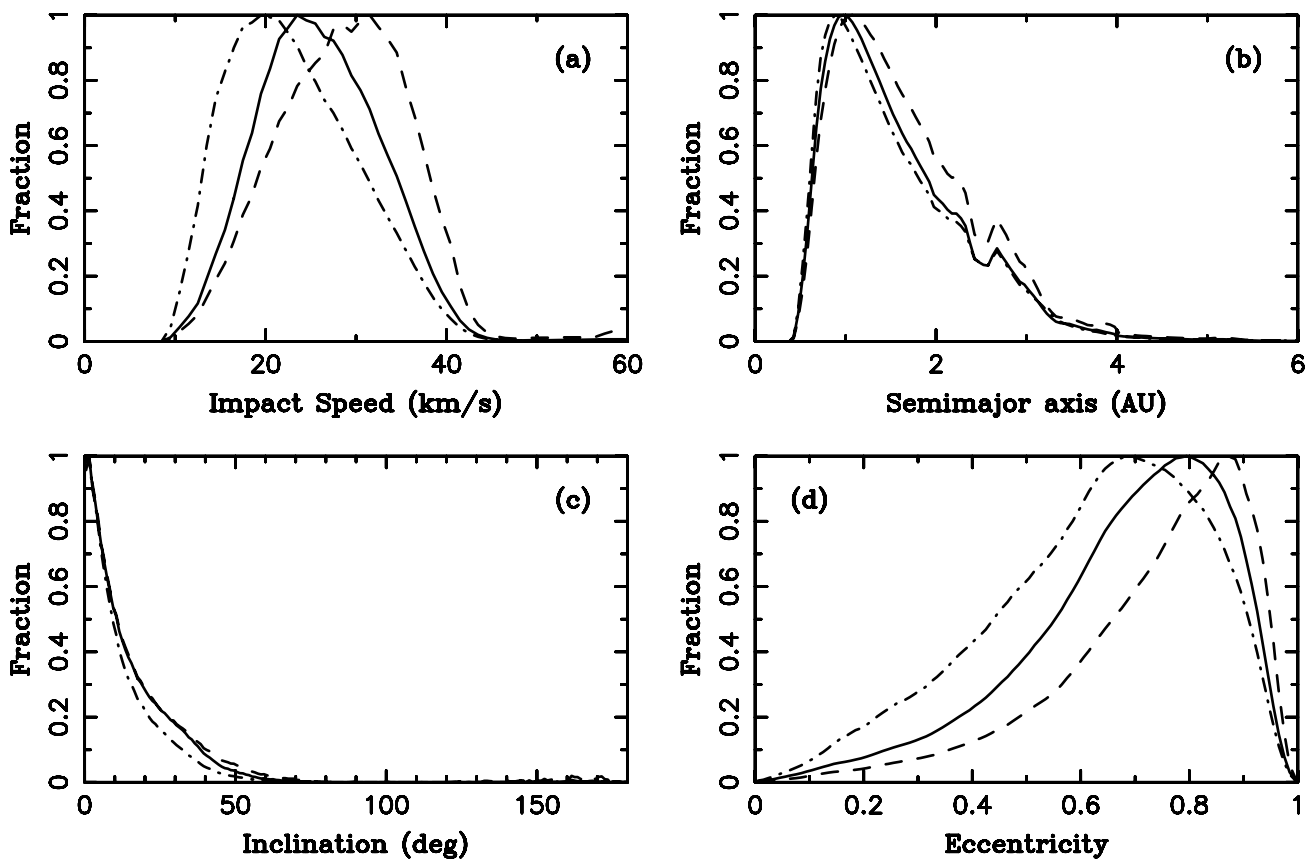

Figure 7. Effect of $D^{*}$. Different lines show the results for $D^{*}=100 \mu \mathrm{m}$ (solid), $D^{*}=30 \mu \mathrm{m}$ (dashed), and $D^{*}=300 \mu \mathrm{m}$ (dot-dashed). As in Figure 5 , we used $I^{*}=0.003, \alpha_{1}=2, \alpha_{2}=5$, and $S=1$. No radiant cutoff was applied here.

orbital histories, and thus also a larger spread in $v$. Figure 9 illustrates these trends.

By experimenting with different SFDs, we found that the best matches to AMOR observations can be obtained with $D^{*} \lesssim 50 \mu \mathrm{m}$ and $\alpha_{2}=3.5$, or with $D^{*} \gtrsim 200 \mu \mathrm{m}$ and $\alpha_{1}=3.5$, while the values of $\gamma$ and $S$ are essentially unconstrained (but see Section 3.2 for a discussion of the collisional model). We opt for $D^{*}=200 \mu \mathrm{m}$ in Figure 10, which illustrates one of our preferred models, because the original interpretation of spacecraft impact experiments indicates a change of slope at $D \simeq 200 \mu$ m (e.g., G85; Love \& Brownlee 1993). The solutions with $D^{*} \lesssim 50 \mu \mathrm{m}$ and $\alpha_{2}=3.5$, which would better correspond to the reinterpretation of the impact experiments by Mathews et al. (2001), are also plausible. We will discuss this issue in Section 4.

While our models $d N(v), d N(a)$, and $d N(e)$ in Figure 10 match observations reasonably well, the model $d N(i)$ is slightly narrower than the one measured by AMOR. This indicates that we may be missing sources with larger inclinations. For example, as discussed in Section 2.1, our model for the initial inclinations of JFC particles can be inappropriate if JFCs lose mass gradually by recurrent splitting events (e.g., LD97; Di Sisto 

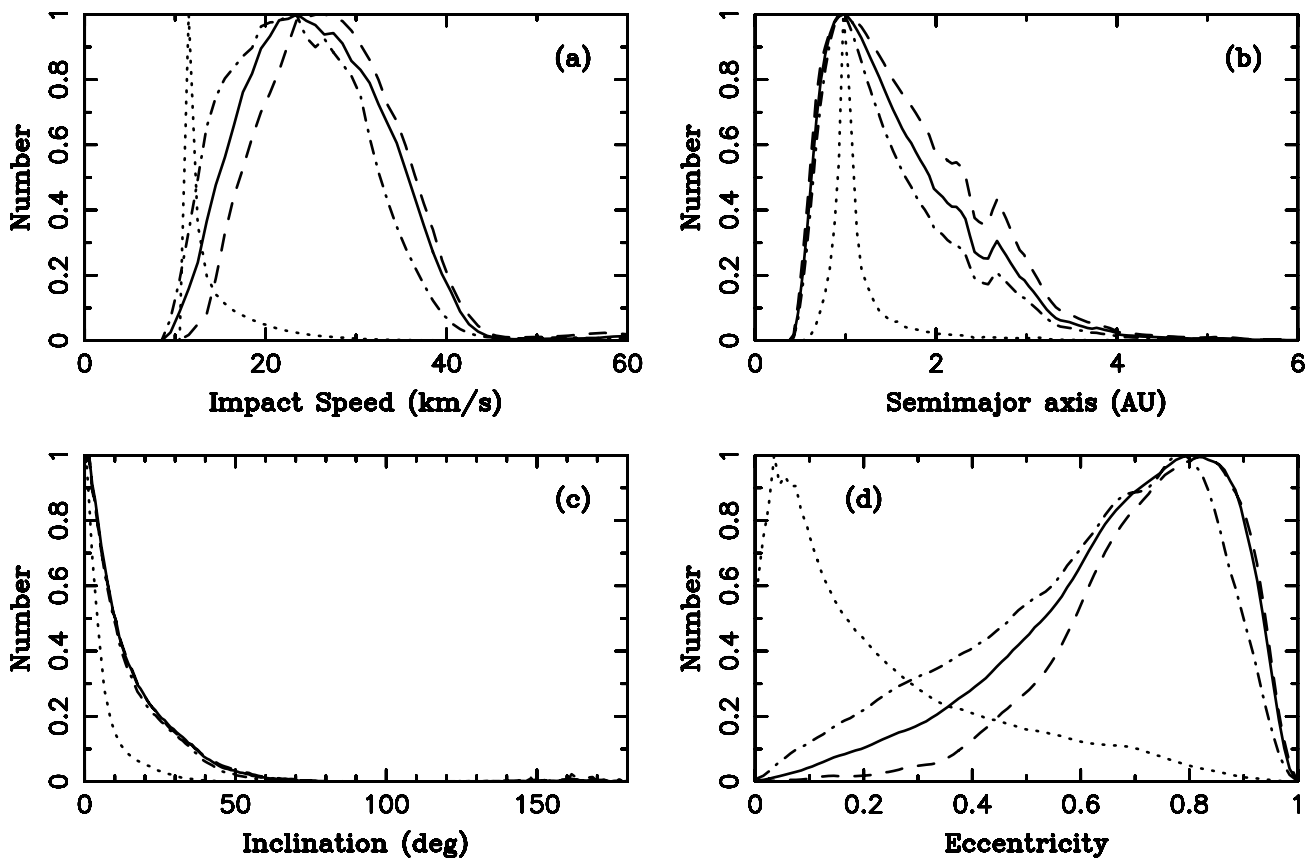

Figure 8. Same as Figure 4 but for $\alpha_{1}=\alpha_{2}=3.5$.
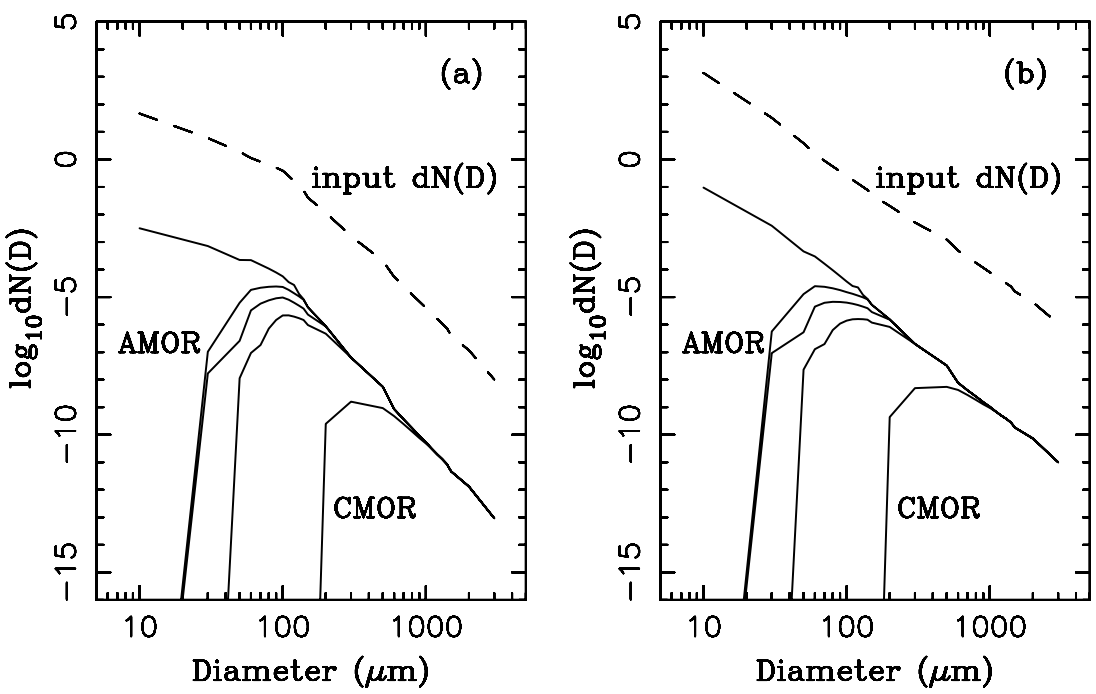

Figure 9. SFDs of different populations for (a) $D^{*}=100 \mu \mathrm{m}, \alpha_{1}=2, \alpha_{2}=5$ and (b) $\alpha_{1}=\alpha_{2}=3.5$. The input distributions, shown by dashed lines, correspond to those used in Figures 4 and 8 . They were normalized to 1 particle with $D>100 \mu \mathrm{m}$. The upper solid line in each panel shows the SFD of particles accreted by the Earth $\left(I^{*}=0\right)$. Accreted particles show a slightly steeper slope than the input SFD for $D \gtrsim 100 \mu \mathrm{m}$, because of the effects of disruptive collisions, which eliminate large particles in the G85 model, and a shallower slope for $D \lesssim 100 \mu \mathrm{m}$, because small JFC particles have smaller Earth-accretion probabilities than the larger ones due to their shorter P-R drag lifetimes. The other solid lines show the expected meteor SFD for AMOR (three lines corresponding, from left to right, to $I^{*}=0.001$, 0.003 , and 0.01$)$ and $\operatorname{CMOR}\left(I^{*}=1\right)$.

et al. 2009; N10). It is also possible, however, that the radiant cutoff of Galligan \& Baggaley $(2004,2005)$ is not sufficiently restrictive to pick up JFC meteoroids only, as hinted on by Figure 1(c), where $d N(i)$ seems to follow different trends for $i<30^{\circ}$ and $i>30^{\circ}$. Note that Campbell-Brown (2008), using a more restrictive radiant cutoff, obtained a relatively narrow $d N(i)$ of helion/antihelion meteors.

Figure 11 shows the radiant distributions for our preferred model shown in Figure 10. With $I^{*}=0$, the radiants fill the whole sky and show broad concentrations around $l=-90^{\circ}$, $l=90^{\circ}$, and $b=0^{\circ}$. With $I^{*}=0.003$, however, the radiants become tightly clustered about $l=-70^{\circ}, l=70^{\circ}$, and $b=0^{\circ}$. This highlights the importance of the ionization cutoff. For a comparison, Galligan \& Baggaley (2005) found that the helion and antihelion sources are centered at $l= \pm 70^{\circ}$ and their full widths are $\simeq 20^{\circ}$ in both $l$ and $b$. The location and spread of our model radiants very closely match these measurements.

\section{2. $C M O R$}

The results discussed in Section 3.1 were obtained with the standard G85 model for the collisional disruption of particles. In G85, the large, millimeter-sized particles have very short physical lifetimes $\left(\sim 10^{4} \mathrm{yr}\right)$ and disrupt before they can significantly evolve by P-R drag. Small particles, on the other hand, have long collisional lifetimes and evolve faster by P-R drag. The G85 model therefore implies different orbital histories of small and large particles and, as we found here, produces significantly 

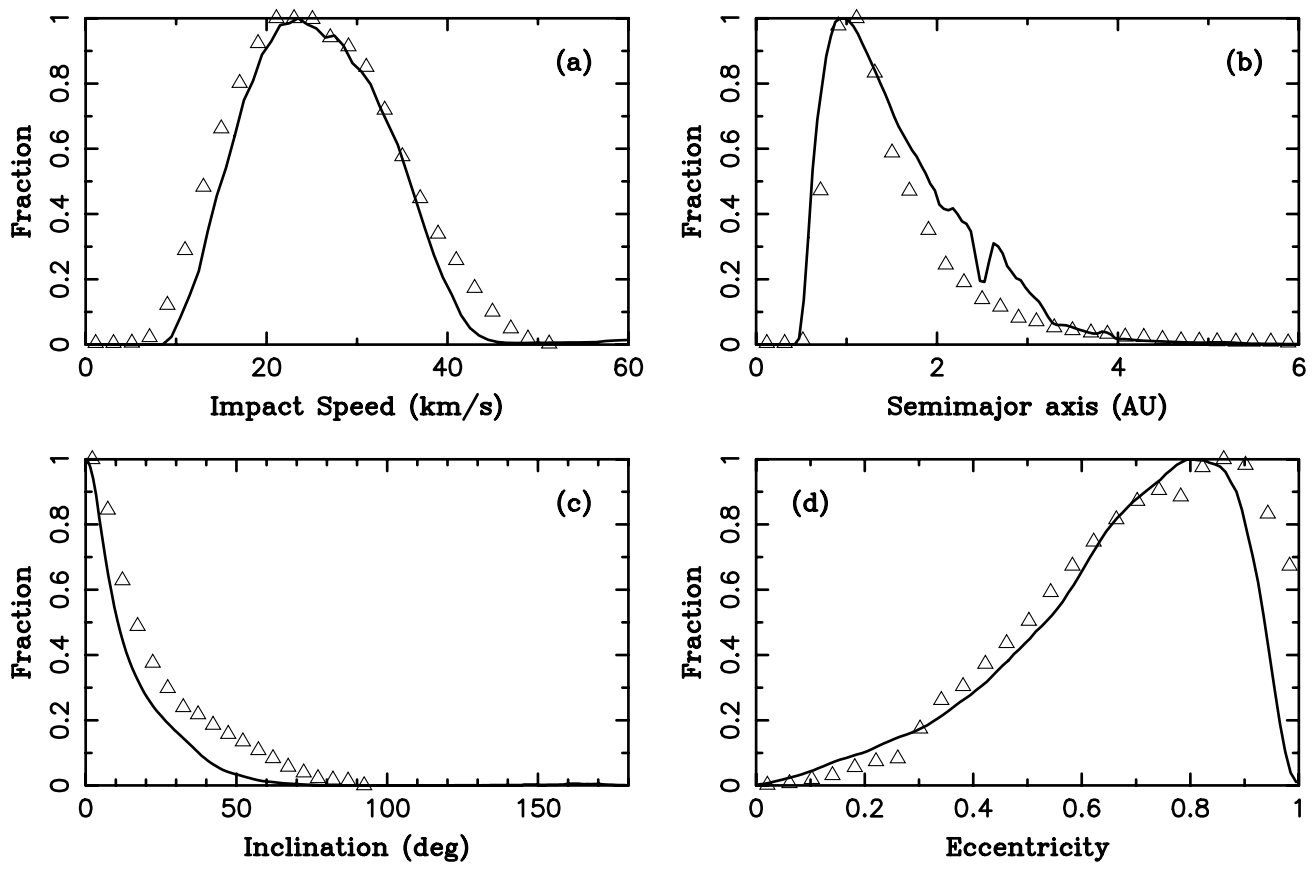

Figure 10. Our preferred model for the AMOR meteors. The triangles label the corrected AMOR data from Galligan \& Baggaley (2005). Solid lines show our results obtained with $I^{*}=0.003, D^{*}=200 \mu \mathrm{m}, \alpha_{1}=3.5, \alpha_{2}=5.0, \gamma=0$, and $S=1$.
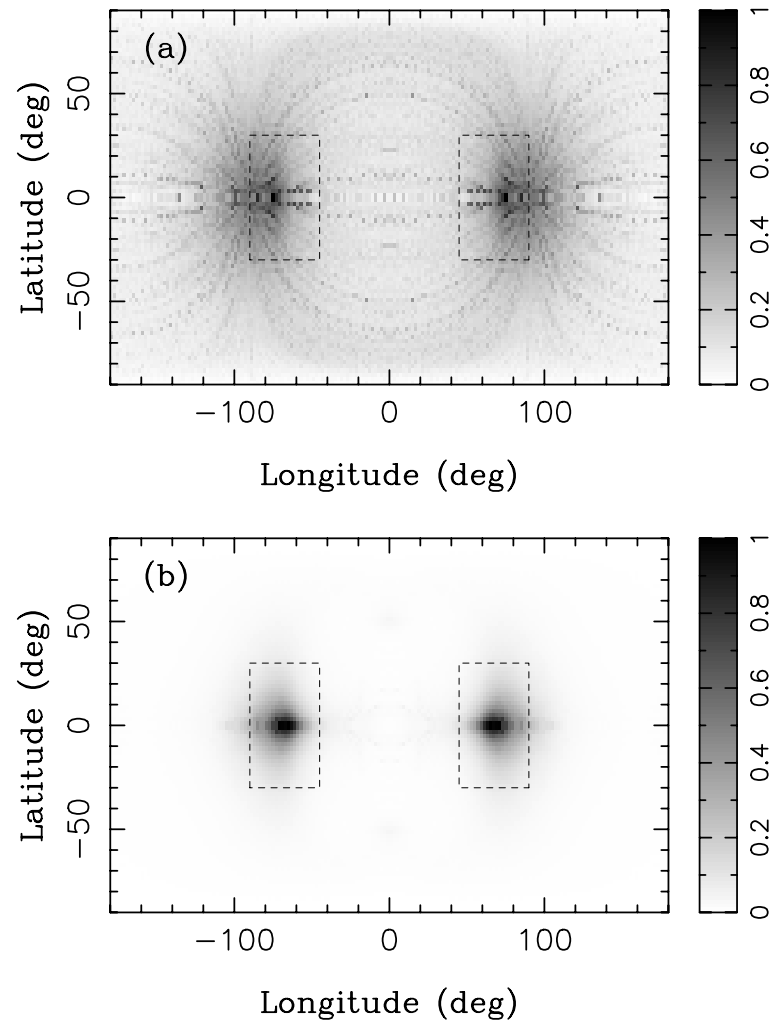

Figure 11. Radiants for $I^{*}=0$ (a) and $I^{*}=0.003$ (b). The model parameters used here are the same as in Figure 10. The gray scale shows the radiant density that was normalized to 1 at its maximum. The dashed rectangles in both panels show our radiant selection criteria defined in Section 2.6.

different distributions of impact speeds and heliocentric orbits for $I^{*}=0.003$ and $I^{*}=1$. Figure 12 illustrates the case of $I^{*}=1$. These results are at odds with observations, because the distributions measured by AMOR and CMOR are not that different.
To resolve this problem, we needed to assume that $\tau_{\text {coll }}$ for $D \sim 1 \mathrm{~mm}$ is significantly longer than in G85 $(S \gtrsim 30)$. Figure 13 shows the results for $S=100$. The main improvement with respect to Figure 12 is that $d N(a)$ now peaks at $a \sim 1 \mathrm{AU}$. This is a direct consequence of longer $\tau_{\text {coll }}$, which allows the large particles to accumulate larger P-R drifts and reach $a \sim 1$ AU. On the other hand, models with $S \gtrsim 30$ and $I^{*}=0.01-0.001$ do not match the AMOR measurements. This shows that the size dependence of the G85 collisional model may be incorrect. Taken together, if $S \gtrsim 30$ is needed to match CMOR, while $S \sim 1$ is needed to match AMOR, $\tau_{\text {coll }}(D)$ should be more constant over the relevant size range, $D \sim 30-1000 \mu \mathrm{m}$ according to Figure 9, than suggested by G85.

We performed a search in parameter space to see whether we can obtain the impact speed and orbit distributions with $S=100$ that would closely resemble those measured by CMOR (Figure 10- 12 in Campbell-Brown 2008). We found that the model results with $\alpha \sim 2$ work best. With $\alpha \sim 2$, at least locally near $D \sim 500 \mu \mathrm{m}$, which are the most important sizes for CMOR, the model distributions have the characteristic shapes measured by CMOR (Figure 14). When no radiant cutoff is used, $d N(v)$ has the maximum just below $v=20 \mathrm{~km} \mathrm{~s}^{-1}$, and $d N(e)$ peaks at $e \sim 0.7$. When the radiant cutoff is applied, $d N(v)$ has the maximum just below $v=30 \mathrm{~km} \mathrm{~s}^{-1}$, and $d N(e)$ peaks at $e \sim 0.8$. These trends correspond very well to those in Figures 10 and 12 in Campbell-Brown (2008).

The model $d N(a)$ with radiant cutoff becomes more tightly clustered at $a \sim 1$ AU than in the case without cutoff, in a nice correspondence to the CMOR measurements (Figure 14(b)). Our $d N(i)$ with radiant cutoff is slightly broader than the CMOR distribution (Figure 14(c)), which is logical because the radiant cutoff used by Campbell-Brown (2008) is more restrictive than the one used here. Overall, the agreement between the model and observations is very good.

While the meteoroid SFD can be wavy (e.g., Ceplecha et al. 1998), with $\alpha \sim 3.5$ at $D \sim 100 \mu \mathrm{m}$ (see Section 3.1) and $\alpha \sim 2$ at $D \sim 500 \mu \mathrm{m}$, these slope determinations can also 

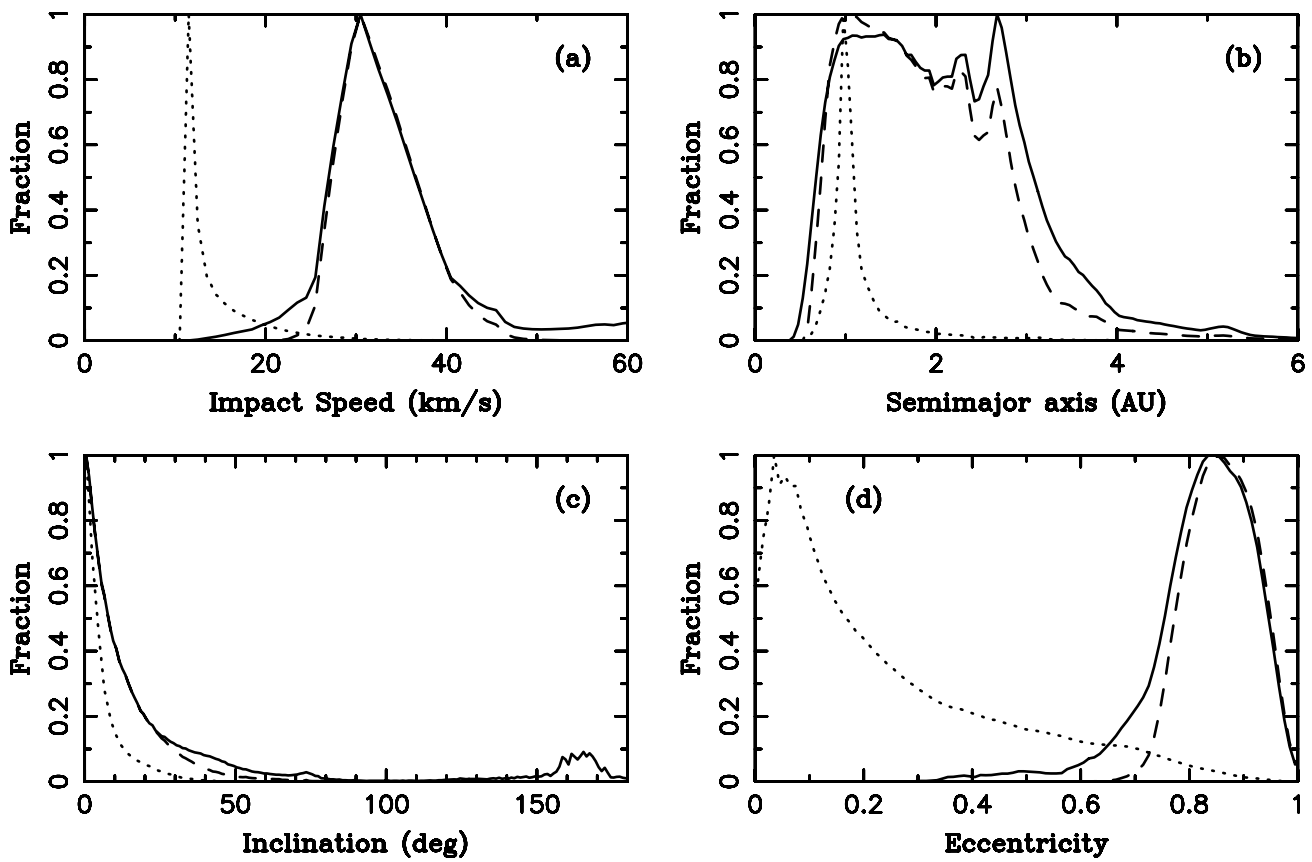

Figure 12. Different lines show the results for $I^{*}=0$ (dotted), $I^{*}=1$ (solid), and $I^{*}=1$ with radiant cutoff (dashed). As in Figure 10, illustrating our preferred fit to the AMOR data, we used $D^{*}=200 \mu \mathrm{m}, \alpha_{1}=3.5, \alpha_{2}=5, \gamma=0$, and $S=1$. The distributions shown here do not match the CMOR observations of the helion/antihelion meteors (cf. Figures 10 and 12 in Campbell-Brown 2008). They may be more similar to the distributions inferred from the visual meteor surveys that are sensitive to larger, $\sim 1 \mathrm{~cm}$ meteoroids (P. Jenniskens et al. 2011, in preparation).
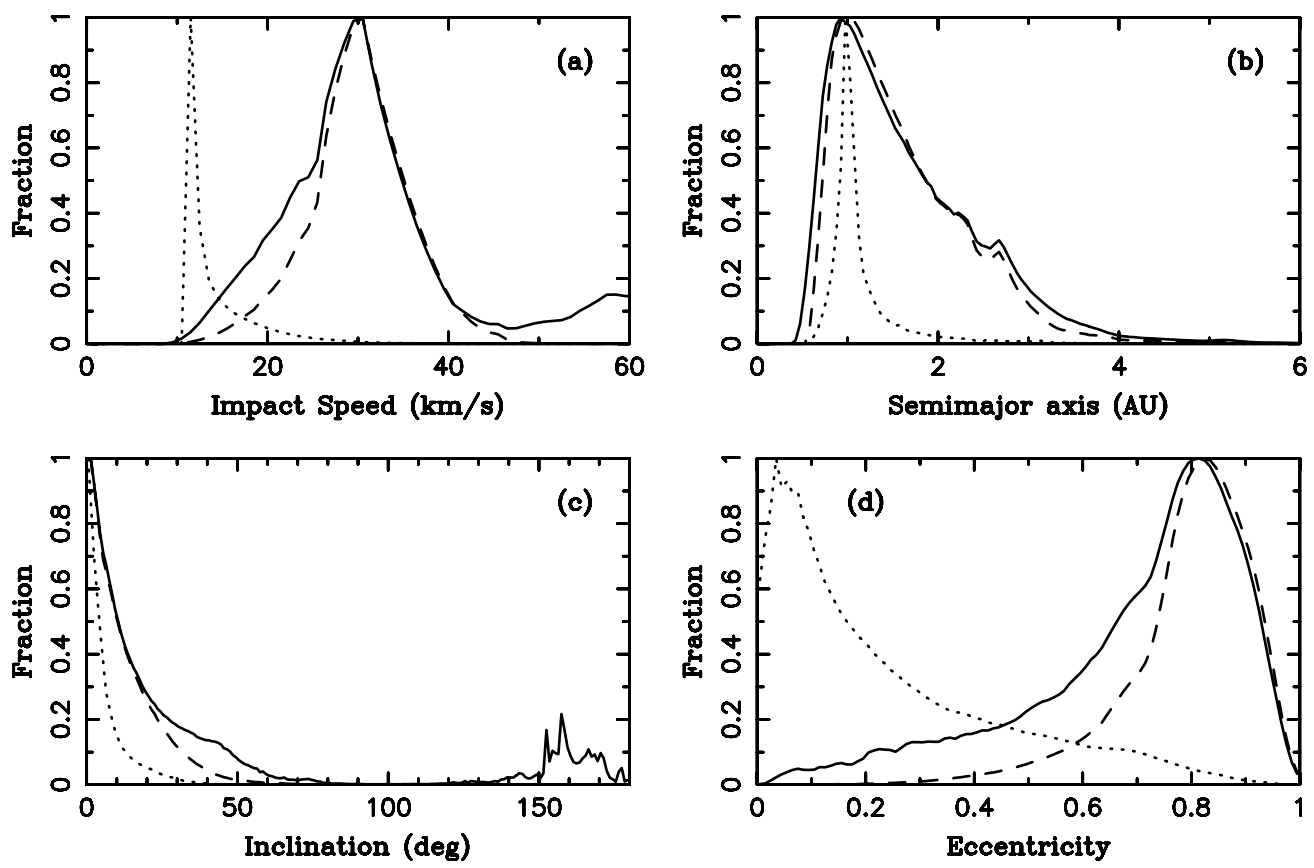

Figure 13. Same as Figure 12 but for $S=100$.

be artificially imposed on the results by our simple treatment of the radar's detection efficiency. ${ }^{13}$ In addition, it is not clear

\footnotetext{
13 The SFD constraints established here were obtained with the simple parameterization of the radar's detection efficiency described in Section 2.6. In reality, the detection efficiency should be a more complex function of the meteoroid mass and speed, other impact parameters, and observing conditions. It is plausible, for example, that the detection probability of a meteor, $D(I)$, goes smoothly from $\sim 0$ for $I \leqslant I_{0}^{*}<I^{*}$ to $\sim 1$ for $I \geqslant I_{1}^{*}>I^{*}$, and attains some intermediate values from $I_{0}^{*}$ to $I_{1}^{*}$. If so, this could broaden the size range of particles that contribute to detections and potentially resolve the problem with the width of $d N(v)$, without the need to resorting to a relatively shallow SFD slope.
}

to us whether it is appropriate to compare our results for the helion/antihelion meteors to Figures 10 and 12 in CampbellBrown (2008), because their Figure 10 shows the CMOR distributions for all sporadic sources, and the distributions in Figure 12 were weighted to a constant limiting mass, a correction that is not needed for a comparison with our model.

Finally, Figure 15 shows the CMOR radiant distributions for models illustrated in Figures 12 and 13. With longer $\tau_{\text {coll }}$, the radiants are slightly more spread around the helion and antihelion directions. Interestingly, Figure 15 indicates that the JFC meteoroids are capable of producing apex meteors. 

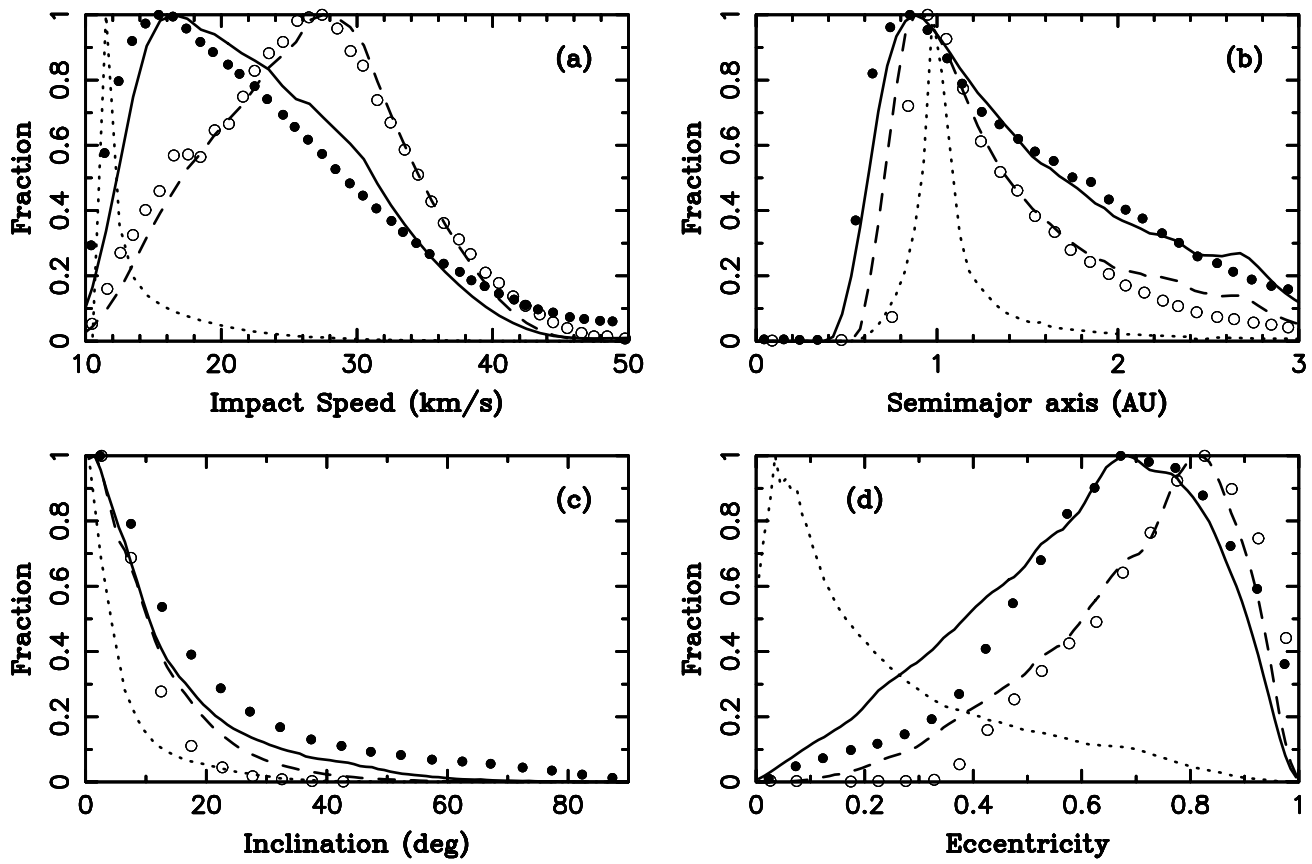

Figure 14. Our preferred model for the CMOR meteors. The filled and unfilled circles label the CMOR data from Figures 10 and 12 in Campbell-Brown (2008). The filled circles are raw CMOR data where no radiant cutoff was applied to separate different sources. The unfilled circles show the distributions, where the antihelion source was isolated by the radiant cutoff defined in Table 1 in Campbell-Brown (2008). These distributions were corrected for the observing biases and mass weighted. The helion meteors, not shown here, have the corrected CMOR distributions very similar to those plotted here. Different lines show the model results for $I^{*}=0$ (dotted), $I^{*}=1$ (solid), and $I^{*}=1$ with radiant cutoff (dashed). We used the same parameters as in Figure 12, except for $S=100$ and $\alpha=2$. These assumed parameters are not inconsistent with those used is Figure 10, because they apply to larger particles. Figures 10 and 14 can therefore be thought as a simultaneous fit to the radar data. Note that the $X$-axis ranges were changed here relative to the previous figures to show things more clearly.
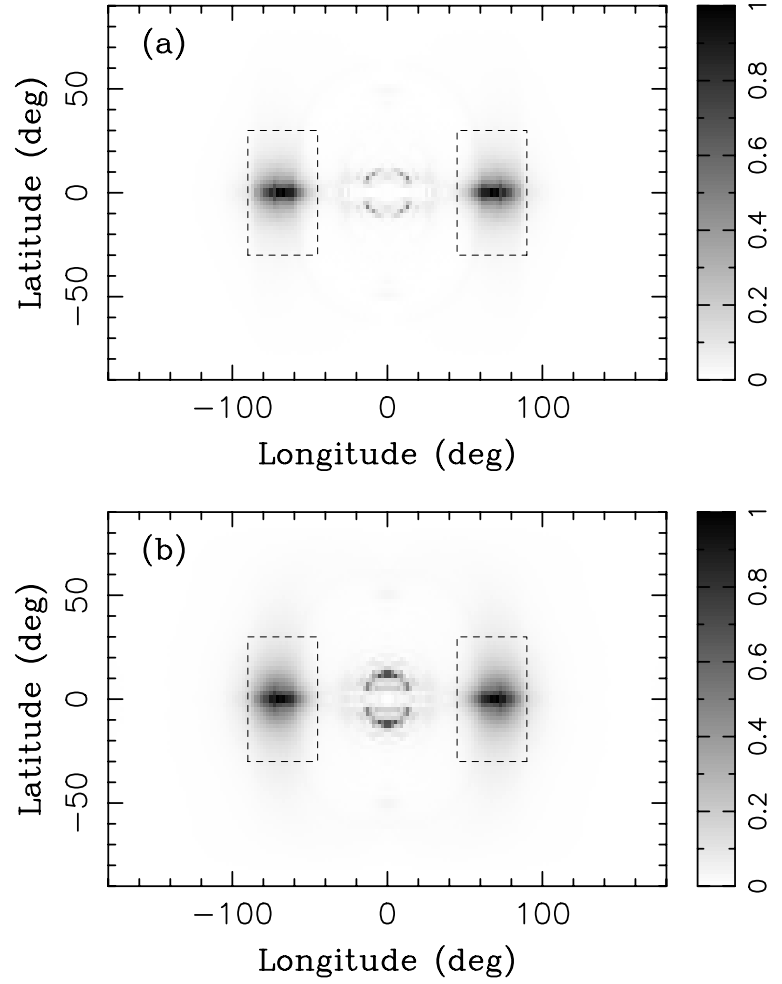

Figure 15. Radiants for $I^{*}=1$ and: (1) $S=1$, and (b) $S=100$. The model parameters used here are the same as in Figures 12 and 13. The gray scale shows the radiant density that was normalized to 1 at its maximum. The dashed rectangles in both panels denote our radiant selection criteria defined in Section 2.6.
These apex particles have prograde orbits, low impact speeds, and very low semimajor axes. They impact from the apex direction because their orbital speed near the aphelion at $1 \mathrm{AU}$ is smaller that the Earth's orbital speed. The contribution of JFC meteoroids to apex meteors should be small, however, relative to those produced by the retrograde $\mathrm{HTC}$ and/or OCC meteoroids. We verified that only a small fraction $(<1 \%)$ of the JFC meteoroids can reach retrograde orbits.

\subsection{IRAS}

Using the methods described in Sections 2.4 and 2.5, we computed the MIR fluxes for all models considered in the previous section. Here we illustrate these results and compare them with those obtained by IRAS. Before we do so, however, we want to emphasize that the $\mathrm{ZC}$ is in all likelihood a mixture of several particle populations, including contribution from asteroids and long-period comets (see, e.g., N10), while here we only model the JFC component. The best fits obtained to IRAS observations in this work are therefore only approximate and could be modified if other components of the $\mathrm{ZC}$ were considered.

Figure 16 shows our results for $D^{*}=100 \mu \mathrm{m}, \alpha_{1}=2$, $\alpha_{2}=5, \gamma=0$, and $S=1$, corresponding to Figures 4 and 5. The model MIR profiles are slightly narrower than the observed ones, but otherwise correspond to IRAS measurements reasonably well. For example, a small, $\lesssim 10 \%$ contribution from a source with a more isotropic distribution of inclinations, such as HTCs and/or OCCs, would easily compensate for the small difference. See N10 for a discussion of additional sources that were not modeled here.

A different way to bring the model and observations into a closer agreement is to assume that $\gamma<0$. With $\gamma<0$, the 


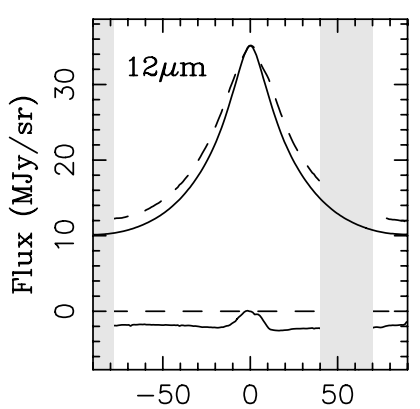

Ecliptic Latitude (deg)

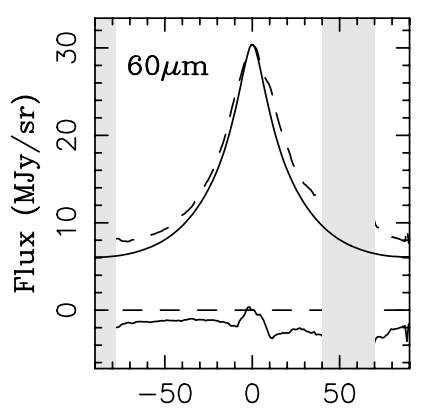

Ecliptic Latitude (deg)

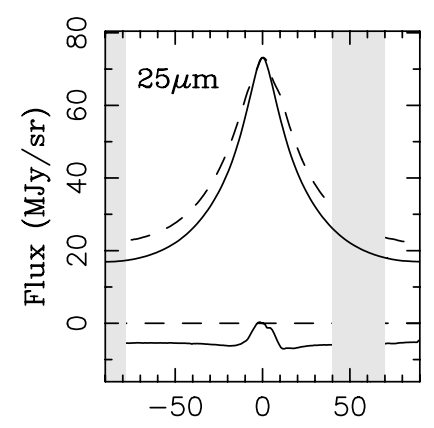

Ecliptic Latitude (deg)

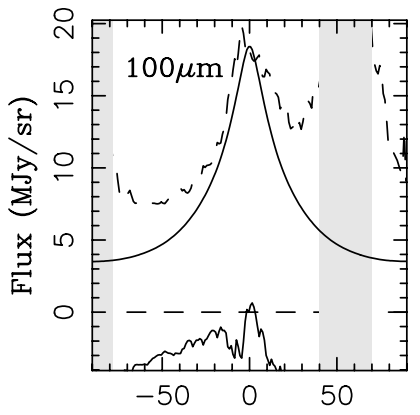

Ecliptic Latitude (deg)

Figure 16. MIR profiles at (a) $12 \mu \mathrm{m}$, (b) $25 \mu \mathrm{m}$, (c) $60 \mu \mathrm{m}$, and (d) $100 \mu \mathrm{m}$ wavelengths. The dashed line shows the mean IRAS profiles for $l_{\odot}=90^{\circ}$. The upper solid curves show the model results for the same wavelength and elongation. The bottom lines show the residual flux obtained by subtracting the model flux from the mean IRAS profile. Here we used the same model parameters as in Figure 4: $D^{*}=100 \mu \mathrm{m}, \alpha_{1}=2, \alpha_{2}=5, \gamma=0$ and $S=1$.

distribution of JFC particles is weighted toward low $R$ and is projected to a wider range of $b$ when observed from $R=1$ AU. If $\gamma>0$, on the other hand, the distribution is weighted toward large $R$ and is seen closer to the ecliptic. We tested a continuous range of $\gamma$ values and found that $\gamma \simeq-1.3$ provided the best match to the IRAS observations (Figure 17).

The effects of additional sources and $\gamma \neq 0$ on the MIR profiles are to some degree degenerate in the IRAS model. They would be difficult to separate, based solely on modeling of the IRAS observations, if we included additional sources in the present work. For example, as discussed above, the MIR profiles become broader, and more similar to the IRAS measurements, if $\gamma<0$ and/or if sources with a more isotropic inclination distribution are included (N10). On the other hand, a small asteroid contribution at the $\sim 5 \%-10 \%$ level (Nesvorný et al. 2006) would produce slightly narrower profiles than those obtained with the JFCs alone (N10). A two-source model with JFCs and asteroids would thus require $\gamma<0$.

The MIR profiles obtained in our model are not overly sensitive to the assumptions on the collisional lifetimes of particles. For example, increasing the collisional lifetime of millimeter-sized particles relative to the G85 model, which may be needed to match CMOR observations (Section 3.2), does not affect the results obtained here, because the ZC's cross section is mainly in $D \lesssim 200 \mu \mathrm{m}$ particles (N10).

In addition, the model profiles are also insensitive to the input SFD of the JFC particles. This is because the JFC particles with $D \lesssim 200 \mu \mathrm{m}$ have $\tau_{\text {coll }}$ that exceeds their P-R drag lifetimes. All these particles therefore have roughly similar orbital histories and produce similar MIR profiles. This explains why we obtain

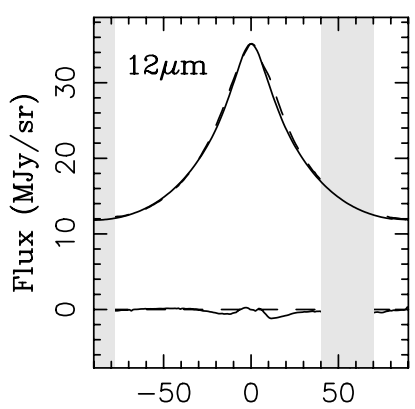

Ecliptic Latitude (deg)

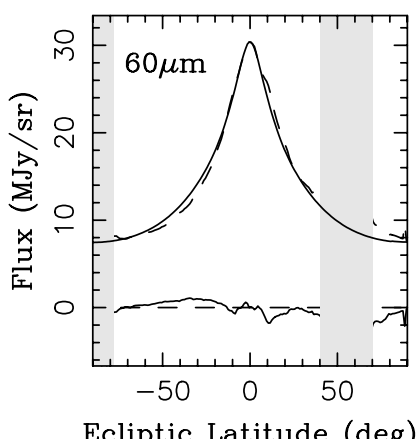

Figure 17. Same as Figure 16, but with $\gamma=-1.3$. The radial distribution obtained with $\gamma=-1.3$ leads to the best fit to IRAS observations, at least for the input SFD assumed here. The MIR profiles, however, are not overly sensitive to the assumed SFD.

nearly identical results for all $D^{*}<200 \mu \mathrm{m} .{ }^{14}$ The effects of $\alpha_{1}$ and $\alpha_{2}$ are also minor.

\subsection{ZC Mass and Cross Section, and Mass Influx on Earth}

The comparison of our model with the IRAS data is important because it allows us to obtain the absolute calibration of the number of particles in the ZC (or, more precisely, their total cross section). This calibration can then be used to estimate the rate of the terrestrial accretion of interplanetary material, both with and without the ionization cutoff, with the former estimate being relevant to radar observations. Note that it is more difficult to derive the overall terrestrial accretion rate from the meteor radar measurements alone, because different radar instruments have different detection sensitivities, and some, such as the less sensitive CMOR, do not detect the very small and/or slow meteoroids (see discussion in Section 4).

Unless we specify otherwise, all estimates quoted below were obtained for the full size range of particles between $D=5 \mu \mathrm{m}$ and $D=1 \mathrm{~cm}\left(10^{-10} \mathrm{~g}\right.$ to $1 \mathrm{~g}$ for $\left.\rho=2 \mathrm{~g} \mathrm{~cm}^{-3}\right)$. These estimates need to be considered with caution because they were obtained with approximate initial SFDs. In reality, the number of particles released by JFCs can be a complicated function of $D$ and should also depend on the circumstances of the splitting/disruption events.

We start by discussing the total cross-section area $\left(\sigma_{\mathrm{ZC}}\right)$ and mass $\left(m_{\mathrm{ZC}}\right)$ of particles in the ZC. For the sake of simplicity, we will assume that $30 \lesssim D^{*} \lesssim 300 \mu \mathrm{m}, \alpha_{1}<3$ and $\alpha_{2}>4$, so that particles with sizes below $30 \mu \mathrm{m}$ and above $300 \mu \mathrm{m}$ do not strongly contribute to the cross section or mass, as suggested

\footnotetext{
14 We note that the model profiles obtained for $\gamma \simeq-1.3$ and $D^{*}=10 \mu \mathrm{m}$ are slightly narrower than those shown in Figure 17 for $D^{*}=100 \mu \mathrm{m}$, because small particles drift faster and their inclinations are disturbed to a lesser degree by planets and planetary resonances.
} 
Table 1

A Summary of Different Models

\begin{tabular}{|c|c|c|c|c|c|c|c|c|c|}
\hline $\begin{array}{l}D^{*} \\
(\mu \mathrm{m})\end{array}$ & $\alpha_{1}$ & $\alpha_{2}$ & $\gamma$ & $\begin{array}{c}\sigma_{\mathrm{ZC}} \\
\left(10^{11} \mathrm{~km}^{2}\right)\end{array}$ & $\begin{array}{c}m_{\mathrm{ZC}} \\
\left(10^{19} \mathrm{~g}\right)\end{array}$ & $\begin{array}{c}\dot{m}_{\mathrm{ZC}} \\
\left(\mathrm{kg} \mathrm{s}^{-1}\right)\end{array}$ & $\begin{array}{c}\dot{m}_{0} \\
\left(\text { tons } \mathrm{yr}^{-1} \text { ) }\right.\end{array}$ & $\begin{array}{c}\dot{m}_{0.003} \\
\left(\text { tons } \mathrm{yr}^{-1} \text { ) }\right.\end{array}$ & $\begin{array}{c}\dot{m}_{1} \\
\text { (tons } \mathrm{yr}^{-1} \text { ) }\end{array}$ \\
\hline 100 & 2 & 5 & 0 & 2.1 & 3.8 & 4200 & 15,000 & 4200 & 240 \\
\hline 100 & 2.9 & 4.1 & 0 & 2.1 & 4.6 & 6200 & 12,000 & 4100 & 860 \\
\hline 100 & 2 & 5 & -1 & 2.0 & 3.8 & 5200 & 15,000 & 6100 & 480 \\
\hline 100 & 2 & 5 & -1.3 & 2.0 & 3.9 & 5800 & 16,000 & 7000 & 590 \\
\hline 100 & 2 & 5 & 1 & 2.3 & 4.0 & 4000 & 14,000 & 3100 & 130 \\
\hline 30 & 2 & 5 & 0 & 1.8 & 1.2 & 1600 & 7700 & 500 & 27 \\
\hline 300 & 2 & 5 & 0 & 3.4 & 15 & 19,000 & 26,000 & 19,000 & 3300 \\
\hline 50 & 2 & 3.5 & 0 & 2.5 & 12 & 25,000 & 18,000 & 9300 & 4100 \\
\hline $50^{*}$ & 2 & 3.5 & 0 & 2.5 & 11 & 13,000 & 17,000 & 8500 & 3300 \\
\hline 200 & 3.5 & 5.0 & 0 & 1.9 & 2.0 & 2400 & 12,000 & 2900 & 230 \\
\hline $200^{*}$ & 3.5 & 5.0 & 0 & 2.1 & 3.0 & 3400 & 11,000 & 2,900 & 220 \\
\hline
\end{tabular}

Notes. See the main text for the definition of parameters shown here. The asterisks denote the cases, where only particles between $D=10 \mu \mathrm{m}$ and $D=3 \mathrm{~mm}$ were considered.

by the spectral observations of the ZC (e.g., Reach et al. 2003), and various other measurements (see, e.g., Ceplecha et al. 1998, and the references therein).

With these assumptions we find that $1.7 \times 10^{11}<\sigma_{\mathrm{ZC}}<$ $3.5 \times 10^{11} \mathrm{~km}^{2}$, where the larger values correspond to $D^{*}=$ $300 \mu \mathrm{m}$. This estimate is in a good agreement with N10 who found that $\sigma_{\mathrm{ZC}}=(2.0 \pm 0.5) \times 10^{11} \mathrm{~km}^{2}$. The uncertainty in $\sigma_{\mathrm{ZC}}$ mainly stems from the uncertainty in $D^{*}$, with $\gamma$ producing only a minor effect. For a reference, the models shown in Figures 16 and 17 have $\sigma_{\mathrm{ZC}}=2.1 \times 10^{11}$ and $2.0 \times 10^{11} \mathrm{~km}^{2}$, respectively (Table 1).

Mass $m_{\mathrm{ZC}}$ is more poorly constrained then $\sigma_{\mathrm{ZC}}$. For $30 \lesssim$ $D^{*} \lesssim 300 \mu \mathrm{m}, \alpha_{1}<3, \alpha_{2}>4$ and $\rho=2 \mathrm{~g} \mathrm{~cm}^{-3}$ we estimate that $10^{19}<m_{\mathrm{ZC}}<1.5 \times 10^{20} \mathrm{~g}$, with larger values corresponding to $D^{*}=300 \mu \mathrm{m}$. For a more restrictive assumption with $D^{*} \simeq 100 \mu \mathrm{m}$, we find that $3 \times 10^{19}<m_{\mathrm{ZC}}<$ $5 \times 10^{19} \mathrm{~g}$, where the exact value depends on $\alpha_{1}, \alpha_{2}$, and $\gamma$. For a reference, the models shown in Figures 16 and 17 have $m_{\mathrm{ZC}}=3.8 \times 10^{19}$ and $3.9 \times 10^{19} \mathrm{~g}$, which roughly corresponds to a $33 \mathrm{~km}$ diameter sphere with $\rho=2 \mathrm{~g} \mathrm{~cm}^{-3}$.

These results compare well with those reported in N10, where it was found that $2.6 \times 10^{19}<m_{\mathrm{ZC}}<5.2 \times 10^{19} \mathrm{~g}$, under the assumption that the continuous SFD can be approximated by a population of the same-size particles with $D=100-200 \mu \mathrm{m}$.

N10 estimated that the input mass rate of $\dot{m}_{\mathrm{ZC}}=$ $1000-1500 \mathrm{~kg} \mathrm{~s}^{-1}$ is needed to keep the ZC in a steady state. Here we obtain larger values, mainly because the population of particles released with low $q$ has shorter lifetimes and needs to be resupplied at a higher rate. If $D^{*} \lesssim 100 \mu \mathrm{m}, \dot{m}_{\mathrm{ZC}}$ ranges between 3000 and $7000 \mathrm{~kg} \mathrm{~s}^{-1}$, with the largest values corresponding to $\gamma=-1.3$ in the model illustrated in Figure 17. Input rate $\dot{m}_{\mathrm{ZC}}$ is also sensitive to $D^{*}$, roughly in the same proportion as $m_{\mathrm{ZC}}$. For example, $\dot{m}_{\mathrm{ZC}} \sim 1600$ and $19,000 \mathrm{~kg} \mathrm{~s}^{-1}$ for $D^{*}=30 \mu \mathrm{m}$ and $D^{*}=300 \mu \mathrm{m}$, respectively. These estimates are valid for $\alpha_{1}<3$ and $\alpha_{2}>4$. The required input rates can be somewhat smaller or larger if $\alpha_{1} \sim 3.5$ and/or $\alpha_{2} \sim 3.5$ (see Table 1).

Finally, we consider the terrestrial accretion rate, $\dot{m}_{I^{*}}$, where $\dot{m}_{I^{*}}$ denotes the rate for $I>I^{*}$. We consider cases with $I^{*}=0, I^{*}=0.003$, and $I^{*}=1$, with the latter two roughly corresponding to our expectations for AMOR and CMOR, respectively. Using the IRAS calibration, we find that our standard model with $D^{*} \simeq 100 \mu \mathrm{m}, \alpha_{1}<3, \alpha_{2}>4$ implies that $\dot{m}_{0}=(15,000 \pm 3000)$ tons $\mathrm{yr}^{-1}, \dot{m}_{0.003}=$

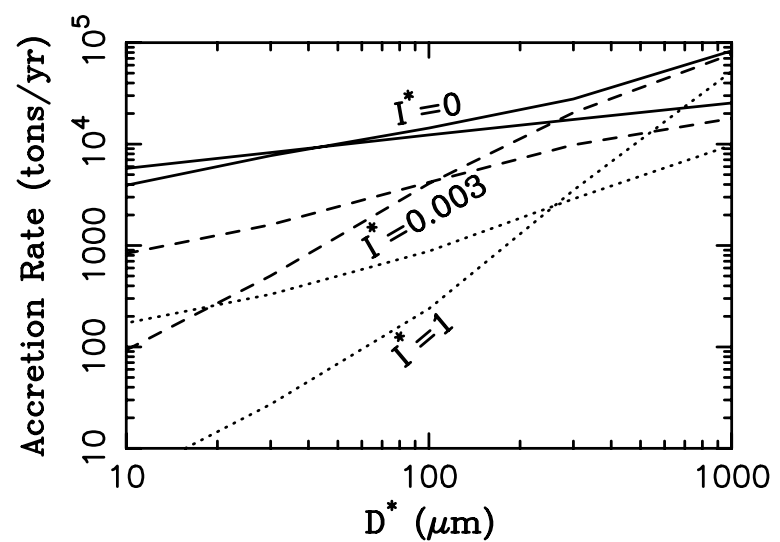

Figure 18. Terrestrial accretion rate of JFC particles as a function of $D^{*}$ obtained in our model for $\gamma=0$ and $S=1$. Different lines denote the results for $I^{*}=0$ (solid), $I^{*}=0.003$ (dashed), and $I^{*}=1$ (dotted). The two lines for each $I^{*}$ were computed for different values of $\alpha_{1}$ and $\alpha_{2}$. The more horizontal lines correspond to $\alpha_{1}=3$ and $\alpha_{2}=4$. The more inclined lines correspond to $\alpha_{1}=2$ and $\alpha_{2}=5$. For $D^{*} \simeq 100 \mu \mathrm{m}$, the overall terrestrial accretion rate for $I^{*}=0$ is $\sim 1-2 \times 10^{4}$ tons $\mathrm{yr}^{-1}$.

(5000 \pm 2000$)$ tons $\mathrm{yr}^{-1}$, and $\dot{m}_{1}=(500 \pm 400)$ tons $\mathrm{yr}^{-1}$, where a large part of the quoted uncertainty comes from the poorly constrained $\gamma$. Note that the values for $I^{*}=0.003$ and $I^{*}=1$ do not include any radiant cutoff.

The uncertainty becomes larger if $D^{*}$ is allowed to vary (Figure 18). For example, with $\alpha_{1}=2, \alpha_{2}=5$, and $\gamma=0$, we obtain $\dot{m}_{0}=26,000$ tons $\mathrm{yr}^{-1}, \dot{m}_{0.003}=19,000$ tons $\mathrm{yr}^{-1}$, and $\dot{m}_{1}=3300$ tons $\mathrm{yr}^{-1}$ for $D^{*}=300 \mu \mathrm{m}$, and $\dot{m}_{0}=$ 7700 tons $\mathrm{yr}^{-1}, \dot{m}_{0.003}=500$ tons $\mathrm{yr}^{-1}$, and $\dot{m}_{1}=27$ tons $\mathrm{yr}^{-1}$ for $D^{*}=30 \mu \mathrm{m}$. Also, our preferred model for the AMOR meteors illustrated in Figure 10 gives $\dot{m}_{0}=12,000$ tons $\mathrm{yr}^{-1}$, $\dot{m}_{0.003}=2900$ tons $\mathrm{yr}^{-1}$, and $\dot{m}_{1}=230$ tons $\mathrm{yr}^{-1}$. Similarly, the model with $D^{*}=50 \mu \mathrm{m}, \alpha_{1}=2$, and $\alpha_{2}=3.5$ gives $\dot{m}_{0}=18,000$ tons $\mathrm{yr}^{-1}, \dot{m}_{0.003}=9300$ tons $\mathrm{yr}^{-1}$, and $\dot{m}_{1}=4100$, or $\dot{m}_{0}=17,000$ tons $\mathrm{yr}^{-1}, \dot{m}_{0.003}=8500$ tons yr$^{-1}$, and $\dot{m}_{1}=3300$, tons $\mathrm{yr}^{-1}$, if the size range of the contributing particles is restricted to $10<D<3000 \mu \mathrm{m}$ (Table 1).

The above estimates with $I^{*}=0$ are a factor of several lower than those found by N10. This difference probably stems from some of the crude approximations used by N10. For example, N10 did not use a continuous SFD of particles and 
approximated $d N(D)$ by delta functions. Their initial particle orbits had (almost exclusively) $q>1.5 \mathrm{AU}$, and did not take into account the fact that many JFCs can split and/or disrupt with $q<1.5$ AU. Moreover, N10 did not properly include the collisional lifetimes of JFC particles in their model. The results presented here, which include all these components, and which were validated on meteor observations, can be more trusted and should supersede those reported in N10.

\section{DISCUSSION}

The results reported in Section 3.4 show that the low-sensitive meteor radars such as CMOR can only detect a few percent of the overall mass flux. It may therefore be difficult to estimate the terrestrial accretion rate from these measurements alone. The more sensitive meteor radars such as AMOR, on the other hand, should detect $10 \%-50 \%$ of the flux, with the exact value mainly depending on the SFD assumptions (Table 1). These more sensitive measurements, especially those obtained with the HPLA radars, are therefore better suited for estimating the overall accretion rate.

The terrestrial accretion rate found here is comparable to that originally inferred by Love \& Brownlee (1993) from the Long Term Duration Facility (LDEF) experiment, and much larger than the one suggested by Mathews et al. (2001) from the Arecibo Observatory (AO) measurements of meteor fluxes. As pointed out by Mathews et al. (2001), the difference between the AO and LDEF measurements could be resolved if LDEF data were recalibrated to $v \sim 50 \mathrm{~km} \mathrm{~s}^{-1}$, which is the prevailing meteor speed as seen at Arecibo (see Janches et al. 2003, 2006; Fentzke et al. 2009).

We found that $d N(v)$ for $I^{*}=0$ peaks at $v \sim v_{\text {esc }}=$ $11.2 \mathrm{~km} \mathrm{~s}^{-1}$ (see, e.g., Figures 4, 8, and 10). The only parameter choices that we were able to identify, where this was not the case, were those where it was assumed that essentially all dust was produced with $q \lesssim 0.5 \mathrm{AU}$, and that $D^{*} \gtrsim 100 \mu \mathrm{m}$ for $S=1$. Such an extreme $q$ dependence seems unlikely, because not many solar system objects-potential parent bodies of meteoroids-ever reach $q \lesssim 0.5 \mathrm{AU}$. (Comet $2 \mathrm{P} /$ Encke has $q=0.34 \mathrm{AU}$, but as we discussed in the Section 1 , the measured mass loss in comet $2 \mathrm{P} /$ Encke is far too low to be dominant.)

The case described above could potentially be interesting, because it could help to explain why the meteor observations at $\mathrm{AO}$, albeit being much more sensitive than AMOR, do not detect a significant population of meteors with $v<15 \mathrm{~km} \mathrm{~s}^{-1}$ (e.g., Janches et al. 2008). To allow for $D^{*}<100 \mu \mathrm{m}$ in this case, and bring our results to a closer agreement with Mathews et al. (2001), $\tau_{\text {coll }}$ of $D<100 \mu \mathrm{m}$ particles, mainly for orbits with $q \lesssim 0.5 \mathrm{AU}$, would need to be significantly shorter than suggested by G85.

To match the CMOR measurements in our model, the collisional lifetime of meteoroids with $D \sim 1 \mathrm{~mm}$ needs to be significantly longer than suggested by G85. Such a long lifetime, of order of a few times $10^{5} \mathrm{yr}$ for a circular orbit at $1 \mathrm{AU}$, can be difficult to reconcile with the inferred lifetimes of meteor streams that seem to disappear on a much shorter timescale (less than a few thousand years; e.g., Jenniskens 2008). Possibly, the centimeter-sized particles released from JFCs, which appear to be dominant in the visual observations of the meteor streams, are physically weak and disrupt in a few thousand years. They could produce a population of millimeter-sized and smaller particles that, according to our work, could be more resistant to collisions.
As explained in Section 2.3, our model neglects small fragments produced by disruptions of larger particles, because it is difficult to account for numerous debris particles in the $N$-body code. Since the fragments are small and should be released on the orbits already evolved by P-R drag, we may speculate that this could lead to a steeper SFD of particles with low perihelion distances. To compensate for that, our preferred model for the source population of particles would need to be adjusted. It is unclear, however, if the effect of the collisional cascade is important. Future work will need to address this problem.

\section{CONCLUSIONS}

The radar observations of sporadic meteors reveal an important population of meteoroids that impact Earth from the helion and antihelion directions. Typically, these particles have heliocentric orbits with $a \sim 1 \mathrm{AU}, e>0.3, i<30^{\circ}$, and dive into the upper atmosphere at speeds $v \sim 20-30 \mathrm{~km} \mathrm{~s}^{-1}$. These results were seemingly inconsistent with the model of the circumsolar meteoroid complex developed in N10, which has been calibrated on the IRAS's MIR observations of the ZC's thermal emission.

The N10 model implied that particles impacting Earth from the helion/antihelion directions should either have $a \sim 1 \mathrm{AU}$ and $e \lesssim 0.3$, or $a \sim 2-4 \mathrm{AU}$ and $e \gtrsim 0.6$. The former case corresponds to $D \lesssim 100 \mu \mathrm{m}$ meteoroids, whose orbits evolved by P-R drag. The latter cases are the large, $D \gtrsim 1 \mathrm{~mm}$ particles that were assumed in N10 to be collisionally disrupted before their orbits could significantly evolve by P-R drag. The different orbital histories of small and large meteoroids in the N10 model would mean that the meteor radars with different detection thresholds should measure very different distributions of the impact speeds and orbits. This is not the case.

Here we showed that the above problem can be resolved if (1) the N10 model is modified to account for the detection efficiency of meteor radars, (2) meteoroids are released from JFCs over a range of perihelion distances with at least some fraction initially having $q \lesssim 1 \mathrm{AU}$, and (3) $D \sim 1 \mathrm{~mm}$ particles have significantly longer ( $\gtrsim 30$ times) collisional lifetimes than those estimated in G85. With these modifications of the N10 model, the results match meteor constraints.

We also found, using the AMOR observations as a constraint, that $D \sim 100-\mu \mathrm{m}$ particles cannot have much longer collisional lifetimes than proposed in G85. Together with (3), these results therefore suggest that $D \sim 100 \mu \mathrm{m}$ and $D \sim 1 \mathrm{~mm}$ meteoroids may have more comparable collisional lifetimes (a few times $10^{5} \mathrm{yr}$ for a circular orbit at $1 \mathrm{AU}$ ) than thought before. If so, the SFD shape inferred from the measurements of the spacecraft impact detectors (such as, e.g., Ulysses, Galileo, LDEF; G85, Love \& Brownlee 1993) may be more closely related to the initial SFD of particles released at sources then to the collisional destruction of particles in space.

We showed that the modified N10 model can successfully match the telescopic observations of the ZC. Using IRAS to calibrate the model, we estimated that the cross section and mass of the $\mathrm{ZC}$ are $\sigma_{\mathrm{ZC}}=(1.7-3.5) \times 10^{11} \mathrm{~km}^{2}$ and $m_{\mathrm{ZC}} \sim 4 \times 10^{19} \mathrm{~g}$. The terrestrial accretion rate of JFC particles was found to be $\sim 15,000$ tons $\mathrm{yr}^{-1}$, of which only a few percent should be detected by CMOR, and 10-50\% should be detected by the more sensitive AMOR.

Moreover, some $10^{3}-10^{4} \mathrm{~kg} \mathrm{~s}^{-1}$ of material must be provided by JFCs to keep the ZC in a steady state. This new input mass estimate is up to $\sim 10$ times larger than the one suggested by N10 (see also Leinert et al. 1983), because particles starting 
with low $q$ have shorter lifetimes, and need to be resupplied at a faster rate. This new estimate resonates with the N10 model in which the $\mathrm{ZC}$ is dominated by the meteoroids released by disrupting/splitting JFCs, because the observed activity of JFCs cannot provide the needed input.

This article is based on work supported by the NASA's Planetary Geology and Geophysics and Planetary Astronomy programs. D.J.'s participation in this work was supported through the NSF Award AST 0908118. The work of D.V. and P.P. was partially supported by the Czech Grant Agency (grants 205/08/0064 and 205/08/H005) and the Research Program MSM0021620860 of the Czech Ministry of Education. We thank Margaret Campbell-Brown, Jack Baggaley, and Hal Levison for useful discussions. We also thank the anonymous reviewer for excellent suggestions to this work.

\section{REFERENCES}

Boehnhardt, H., Tozzi, G. P., Bagnulo, S., et al. 2008, A\&A, 489, 1337 Bottke, W. F., Nolan, M. C., Greenberg, R., \& Kolvoord, R. A. 1994, Icarus, 107,255

Burns, J. A., Lamy, P. L., \& Soter, S. 1979, Icarus, 40, 1

Campbell-Brown, M. D. 2008, Icarus, 196, 144

Ceplecha, Z., Borovička, J., Elford, W. G., et al. 1998, Space Sci. Rev., 84, 327

Dermott, S. F., Jayaraman, S., Xu, Y. L., Gustafson, B. Å. S., \& Liou, J. C. 1994, Nature, 369, 719

Dikarev, V., Grün, E., Baggaley, J., et al. 2005, Adv. Space Res., 35, 1282

di Sisto, R. P., Fernández, J. A., \& Brunini, A. 2009, Icarus, 203, 140

Drolshagen, G., Dikarev, V., Landgraf, M., Krag, H., \& Kuiper, W. 2008, Earth Moon Planets, 102, 191

Fentzke, J. T., \& Janches, D. 2008, J. Geophys. Res. (Space Physics), 113, 3304

Fentzke, J. T., Janches, D., \& Sparks, J. J. 2009, J. Atmos. Sol.-Terr. Phys., 71, 653

Fernández, J. A. 2005, in Comets: Nature, Dynamics, Origin and Their Cosmogenic Relevance (Dordrecht: Springer), 249

Fernández, Y. R., Lisse, C. M., Käufl, H. U., et al. 2000, Icarus, 147, 145

Galligan, D. P., \& Baggaley, W. J. 2004, MNRAS, 353, 422
Galligan, D. P., \& Baggaley, W. J. 2005, MNRAS, 359, 551

Greenberg, R. 1982, AJ, 87, 184

Grun, E., Zook, H. A., Fechtig, H., \& Giese, R. H. 1985, Icarus, 62, 244 (G85)

Hauser, M. G., Gillett, F. C., Low, F. J., et al. 1984, ApJ, 278, L15

Henning, T., \& Mutschke, H. 1997, A\&A, 327, 743

Janches, D., Nolan, M. C., Meisel, D. D., et al. 2003, J. Geophys. Res. (Space Physics), 108, 1222

Janches, D., Heinselman, C. J., Chau, J. L., Chandran, A., \& Woodman, R. 2006, J. Geophys. Res. (Space Physics), 111, 7317

Janches, D., Close, S., \& Fentzke, J. T. 2008, Icarus, 193, 105

Jenniskens, P. 2008, Earth Moon Planets, 102, 505

Jones, J., \& Brown, P. 1993, MNRAS, 265, 524

Jones, J., Campbell, M., \& Nikolova, S. 2001, Meteoroids 2001 Conf., 495, 575

Kelsall, T., Weiland, J. L., Franz, B. A., et al. 1998, ApJ, 508, 44

Kessler, D. J. 1981, Icarus, 48, 39

Kessler-Silacci, J. E., Dullemond, C. P., Augereau, J.-C., et al. 2007, ApJ, 659 , 680

Leinert, C., Roser, S., \& Buitrago, J. 1983, A\&A, 118, 345

Levison, H. F., \& Duncan, M. J. 1994, Icarus, 108, 18

Levison, H. F., \& Duncan, M. J. 1997, Icarus, 127, 13 (LD97)

Love, S. G., \& Brownlee, D. E. 1993, Science, 262, 550

Mathews, J. D., Janches, D., Meisel, D. D., \& Zhou, Q.-H. 2001, Geophys. Res. Lett., 28, 1929

Moro-Martín, A., \& Malhotra, R. 2002, AJ, 124, 2305

Nesvorný, D., Vokrouhlický, D., Bottke, W. F., \& Sykes, M. 2006, Icarus, 181, 107

Nesvorný, D., Jenniskens, P., Levison, H. F., et al. 2010, ApJ, 713, 816 (N10)

Nesvorný, D., Vokrouhlický, D., Pokorný, P., \& Janches, D. 2011, ApJ, in press Öpik, E. J. 1951, Proc. R. Irish Acad. Sect. A, 54, 165

Reach, W. T., Morris, P., Boulanger, F., \& Okumura, K. 2003, Icarus, 164, 384

Reach, W. T., Kelley, M. S., \& Sykes, M. V. 2007, Icarus, 191, 298

Šidlichovský, M., \& Nesvorný, D. 1994, A\&A, 289, 972

Taylor, A., \& McBride, N. 1997, Second European Conference on Space Debris, 393,375

Valsecchi, G. B. 1999, in IAU Colloq. 173, Evolution and Source Regions of Asteroids and Comets, ed. J. Svoren, E. M. Pittich, \& H. Rickman (Tatranska Lomnica: Astronomical Institute of the Slovak Academy of Sciences), 353

Wetherill, G. W. 1967, J. Geophys. Res., 72, 2429

Whipple, F. L. 1939, Publ. Am. Astron. Soc., 9, 235

Whipple, F. L. 1951, ApJ, 113, 464

Wiegert, P., Vaubaillon, J., \& Campbell-Brown, M. 2009, Icarus, 201, 295(W09)

Wisdom, J., \& Holman, M. 1991, AJ, 102, 1528 